$5-1-2009$

\title{
Optical Stress on the Surface of a Particle: Homogeneous Sphere
}

Feng Xu

James A. Lock

Cleveland State University, j.lock@csuohio.edu

Gérard Gouesbet

Cameron Tropea

Follow this and additional works at: https://engagedscholarship.csuohio.edu/sciphysics_facpub

Part of the Physics Commons

How does access to this work benefit you? Let us know!

Publisher's Statement

Copyright 2009 American Physical Society. Available on publisher's site at http://pra.aps.org/ abstract/PRA/v79/i5/e053808.

\section{Original Citation}

Xu, Feng, James A. Lock, Gerard Gouesbet, and Cameron Tropea. "Optical Stress on the Surface of a Particle: Homogeneous Sphere." Physical Review A 79 (2009): 53808.

\section{Repository Citation}

Xu, Feng; Lock, James A.; Gouesbet, Gérard; and Tropea, Cameron, "Optical Stress on the Surface of a Particle: Homogeneous Sphere" (2009). Physics Faculty Publications. 86.

https://engagedscholarship.csuohio.edu/sciphysics_facpub/86

This Article is brought to you for free and open access by the Physics Department at EngagedScholarship@CSU. It has been accepted for inclusion in Physics Faculty Publications by an authorized administrator of EngagedScholarship@CSU. For more information, please contact library.es@csuohio.edu. 
PHYSICAL REVIEW A 79, 053808 (2009)

\title{
Optical stress on the surface of a particle: Homogeneous sphere
}

\author{
Feng $\mathrm{Xu}^{*}$ \\ Fachgebiet Strömungslehre und Aerodynamik, Technische Universität Darmstadt, Petersenstraße 30, 64289 Darmstadt, Germany \\ James A. Lock \\ Department of Physics, Cleveland State University, Cleveland, Ohio 44115, USA \\ Gérard Gouesbet \\ UMR 6614/CORIA CNRS, Université et INSA de Rouen, Site du Madrillet, Avenue de l'Université, BP 12, \\ 76801 Saint Etienne du Rouvray, France \\ Cameron Tropea \\ Fachgebiet Strömungslehre und Aerodynamik, Technische Universität Darmstadt, Petersenstraße 30, 64289 Darmstadt, Germany
}

(Received 21 January 2009; published 5 May 2009)

\begin{abstract}
A rigorous solution (generalized Lorenz-Mie theory) and an approximate solution (geometrical optics) are developed for the optical stress distribution on the surface of a homogeneous sphere. Demonstration calculations are made for plane-wave and Gaussian-beam illuminations. The influence of diffracted waves, surface waves, interference effects, caustic points, beam width, and refractive index on the stress is analyzed. The Debye series is used to evaluate the validity of geometrical optics and provides a quantitative analysis of the contribution of rays of different orders.
\end{abstract}

DOI: 10.1103/PhysRevA.79.053808

PACS number(s): 42.25.Fx, 87.80.Cc

\section{INTRODUCTION}

The radiation force and torque induced by a laser beam on a particle have been widely utilized for optical trapping or manipulation for the past 30 years. To date, various experimental instruments, e.g., optical tweezers [1], optical stretcher [2], optical micromotor [3,4], and optical aligner [5], have been created to meet specific requirements in various applications in medicine, biology, and physics [6]. Regarding the theoretical aspect, expressions for the optical force and torque in analytical form have been derived within the framework of Lorenz-Mie theory (LMT) and generalized Lorenz-Mie theory (GLMT) [7-11]. LMT was established to describe the interaction of a sphere with plane wave $[7,8]$, while GLMT extended LMT's limit to the case of shaped beam incidence [9-11]. Based on the separation of variable method, similar extensions were derived by Barton et al. [12], Kim and Lee [13], and Chang and Lee [14].

To date, most studies have concentrated on the net force and torque, which determine the overall displacement and rotation of a sphere in the beam. However, when a soft particle, e.g., a droplet or a biological cell, is trapped or manipulated, the interaction between the particle and the beam brings in not only the overall displacement and rotation of the particle, but also its shape deformation. To predict and analyze the shape deformation, the geometrical optics (GO) approximation was applied to obtain an approximate stress profile on a nonabsorbing spherical surface [15]. In this paper, however, we present the GLMT solution and improve the GO solution to the surface stress calculation, which forms the basis for further extension to nonspherical shapes.

*f3_xu@yahoo.com
Further, we compare the results of GO and GLMT and determine the physical interpretation of the various features of the stress profile. The Debye series decomposition of the GLMT results is found to be especially useful in this regard. We also determine the dependence of the stress profile on the beam width and its positioning.

\section{ELECTROMAGNETIC THEORY}

A monochromatic arbitrarily shaped beam propagates parallel to the positive $z$ axis and is incident on a sphere of radius $a$. The electric field is assumed to be linearly polarized in the $x$ direction at the beam waist (see Fig. 1). The timedependent part of its electromagnetic fields is $e^{-i \omega t}$, with $\omega$ being the angular frequency.

\section{A. Electromagnetic field description}

Knowing the coordinates of the beam center $\mathrm{O}_{\mathrm{B}}\left(x_{0}, y_{0}, z_{0}\right)$ in the reference frame of the particle, the incident fields

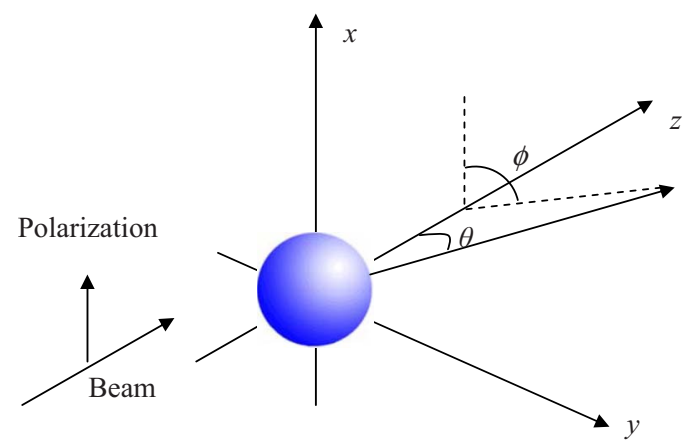

FIG. 1. (Color online) Geometry of a shaped beam incident on a sphere in the Cartesian coordinates $\mathrm{O}_{\mathrm{P}}-x y z$, with the origin defined at the particle center. 
$\left(\mathbf{E}^{(i)}, \mathbf{H}^{(i)}\right)$ can be described exactly in terms of spherical vector wave functions $\left(\mathbf{m}_{m n}, \mathbf{n}_{m n}\right)$ multiplied by a set of the beam shape coefficients $g_{n, T E}^{m}$ and $g_{n, T M}^{m}[16]$ in the spherical coordinate system $(r, \theta, \phi)$ :

$$
\begin{aligned}
\mathbf{E}^{(i)}= & \sum_{m=-\infty}^{\infty} \sum_{n=|m|, n \neq 0}^{\infty} C_{n}^{p w}\left[i g_{n, T E}^{m} \mathbf{m}_{m n}^{(i)}\left(k_{\mathrm{I}} r, \theta, \phi\right)+g_{n, T M}^{m} \mathbf{n}_{m n}^{(i)}\right. \\
& \left.\times\left(k_{\mathrm{I}} r, \theta, \phi\right)\right], \\
\mathbf{H}^{(i)}= & -\frac{i k_{\mathrm{I}}}{\omega \mu_{\mathrm{I}}} \sum_{m=-\infty}^{\infty} \sum_{n=|m|, n \neq 0}^{\infty} C_{n}^{p w}\left[g_{n, T M}^{m} \mathbf{m}_{m n}^{(i)}\left(k_{\mathrm{I}} r, \theta, \phi\right)+i g_{n, T E}^{m} \mathbf{n}_{m n}^{(i)}\right. \\
& \left.\times\left(k_{\mathrm{I}} r, \theta, \phi\right)\right],
\end{aligned}
$$

where $k_{\mathrm{I}}$ is the wave number of the surrounding medium denoted by the subscript I, $\mu_{\mathrm{I}}$ is the permeability of the medium, and the coefficient $C_{n}^{p w}$ is given by $C_{n}^{p w}=i^{n+1}$ $[(2 n+1) / n(n+1)]$.

Likewise, the scattered fields $\left(\mathbf{E}^{(s)}, \mathbf{H}^{(s)}\right)$ can be expressed in terms of the vector wave functions in the following way:

$$
\begin{aligned}
\mathbf{E}^{(s)}= & \sum_{m=-\infty}^{\infty} \sum_{n=|m|, n \neq 0}^{\infty} C_{n}^{p w}\left[i B_{n}^{m} \mathbf{m}_{m n}^{(s)}\left(k_{\mathrm{I}} r, \theta, \phi\right)\right. \\
& \left.+A_{n}^{m} \mathbf{n}_{m n}^{(s)}\left(k_{\mathrm{I}} r, \theta, \phi\right)\right], \\
\mathbf{H}^{(s)}=- & \frac{i k_{\mathrm{I}}}{\omega \mu_{\mathrm{I}}} \sum_{m=-\infty}^{\infty} \sum_{n=|m|, n \neq 0}^{\infty} C_{n}^{p w}\left[A_{n}^{m} \mathbf{m}_{m n}^{(s)}\left(k_{\mathrm{I}} r, \theta, \phi\right)\right. \\
& \left.+i B_{n}^{m} \mathbf{n}_{m n}^{(s)}\left(k_{\mathrm{I}} r, \theta, \phi\right)\right] .
\end{aligned}
$$

The internal fields $\left(\mathbf{E}^{(t)}, \mathbf{H}^{(t)}\right)$ can be described by

$$
\begin{aligned}
\mathbf{E}^{(t)}= & \sum_{m=-\infty}^{\infty} \sum_{n=|m|, n \neq 0}^{\infty} C_{n}^{p w}\left[i D_{n}^{m} \mathbf{m}_{m n}^{(t)}\left(k_{\mathrm{II}} r, \theta, \phi\right)\right. \\
& \left.+C_{n}^{m} \mathbf{n}_{m n}^{(t)}\left(k_{\mathrm{II}} r, \theta, \phi\right)\right], \\
\mathbf{H}^{(t)}=- & \frac{i k_{\mathrm{II}}}{\omega \mu_{\mathrm{II}}} \sum_{m=-\infty}^{\infty} \sum_{n=|m|, n \neq 0}^{\infty} C_{n}^{p w}\left[C_{n}^{m} \mathbf{m}_{m n}^{(t)}\left(k_{\mathrm{II}} r, \theta, \phi\right)\right. \\
& \left.+i D_{n}^{m} \mathbf{n}_{m n}^{(t)}\left(k_{\mathrm{II}} r, \theta, \phi\right)\right],
\end{aligned}
$$

where the subscript II indicates the interior of the particle and $k_{\mathrm{II}}$ and $\mu_{\mathrm{II}}$ are the wave number and permeability of the particle, respectively. By solving the equations obtained from the boundary conditions which ensure the continuity of the tangential components of electric and magnetic fields at the surface of the particle $(r=a)$, the unknown coefficients can be determined as $A_{n}^{m}=g_{n, T M}^{m} a_{n}, \quad B_{n}^{m}=g_{n, T E}^{m} b_{n}$ and $C_{n}^{m}$ $=\left(M_{\mathrm{II}} / M_{\mathrm{I}}\right) g_{n, T M}^{m} c_{n}, D_{n}^{m}=\left(M_{\mathrm{II}} / M_{\mathrm{I}}\right)^{2} g_{n, T E}^{m} d_{n}$, where $\left(a_{n}, b_{n}\right)$ and $\left(c_{n}, d_{n}\right)$ are the Mie coefficients for plane-wave incidence [7] and $M_{\mathrm{II}}$ and $M_{\mathrm{I}}$ are the refractive indices of the particle and medium, respectively.

\section{B. Optical stress}

According to conservation of momentum, the radiation force exerted on a particle of arbitrary shape is equal to the average rate at which momentum is conveyed to it. Under steady-state conditions, the stress (or "force density") distribution on the surface of a particle exerted by the electromagnetic fields $(\boldsymbol{E}, \boldsymbol{H})$ can be expressed as the dot product of the unit normal to the particle surface (n) and the time-averaged Maxwell stress tensor $\langle\overleftrightarrow{A}\rangle[17,18]$ :

$$
\boldsymbol{f}=\boldsymbol{n} \cdot\langle\overleftrightarrow{A}\rangle
$$

where

$$
\langle\overleftrightarrow{A}\rangle=\frac{1}{2} \operatorname{Re}\left[\varepsilon \mathbf{E} \mathbf{E}^{*}+\mu \mathbf{H} \mathbf{H}^{*}-\frac{1}{2}\left(\varepsilon E^{2}+\mu H^{2}\right) \overleftrightarrow{I}\right],
$$

$\stackrel{\leftrightarrow}{I}$ is the unit tensor, $\varepsilon$ and $\mu$ designate the permittivity and permeability of the medium, respectively, and the asterisk indicates the complex conjugate.

Assuming both the particle and surrounding medium are linear, isotropic, and nonmagnetic ( $\mu=1$ inside and outside the particle), after much vector algebra, the surface stress $f$ assumes the following form in spherical coordinates $(r, \theta, \phi)$ :

$$
\boldsymbol{f}=\mathbf{e}_{r} \cdot\langle\overleftrightarrow{A}\rangle=\frac{1}{2} \operatorname{Re}\left[\varepsilon E_{r} \mathbf{E}^{*}+H_{r} \mathbf{H}^{*}-\frac{1}{2}\left(\varepsilon E^{2}+H^{2}\right) \mathbf{e}_{r}\right],
$$

where $\mathbf{e}_{r}$ is the unit vector normal to the sphere surface. Equation (9) has the following equivalent form:

$$
\begin{aligned}
f= & \frac{1}{2} \operatorname{Re}\left\{\frac{1}{2}\left[\varepsilon\left(E_{r}^{2}-E_{\theta}^{2}-E_{\phi}^{2}\right)+\mu\left(H_{r}^{2}-H_{\theta}^{2}-H_{\phi}^{2}\right)\right] \mathbf{e}_{r}\right. \\
& \left.+\left(\varepsilon E_{r} E_{\theta}+\mu H_{r} H_{\theta}\right) \mathbf{e}_{\theta}+\left(\varepsilon E_{r} E_{\phi}+\mu H_{r} H_{\phi}\right) \mathbf{e}_{\phi}\right\} .
\end{aligned}
$$

The total stress $\boldsymbol{f}_{\text {surf }}$ on the surface is equal to the algebraic sum of the stress $\boldsymbol{f}^{(w)}$ exerted by the external electromagnetic fields $\left(\mathbf{E}^{(w)}, \mathbf{H}^{(w)}\right)$ and the stress $\boldsymbol{f}^{(t)}$ exerted by the internal fields $\left(\mathbf{E}^{(t)}, \mathbf{H}^{(t)}\right)$ :

$$
\boldsymbol{f}_{\text {surf }}=\boldsymbol{f}^{(w)}+\boldsymbol{f}^{(t)}=\mathbf{e}_{r} \cdot\left(\left\langle\overleftrightarrow{A}^{(w)}\right\rangle-\left\langle\overleftrightarrow{A}^{(t)}\right\rangle\right)_{r=a} .
$$

Note that the stress exerted by the external (internal) fields acts on the external (internal) sides of the surface. The unit normal vector associated with external (internal) surface is outwardly (inwardly) directed. Therefore in Eq. (11) a minus sign appears before the internal Maxwell tensor $\left\langle\leftrightarrow^{(t)}\right\rangle$ when the outward normal $\mathbf{e}_{r}$ to the external surface is used, which is defined to be positive by convention. In addition, the external electric field is equal to the sum of the incident and scattered fields:

$$
\begin{gathered}
E^{(w)}=E^{(i)}+E^{(s)}, \\
H^{(w)}=H^{(i)}+H^{(s)} .
\end{gathered}
$$

Invoking the boundary conditions across the sphere surface, the $\mathbf{e}_{\theta}$ and $\mathbf{e}_{\phi}$ components of the optical stress disappear and the stress exerted on the surface of the particle is found to be simply dependent on the radial component $\mathbf{e}_{r}$ :

$$
\begin{aligned}
f_{\text {surf }}= & \frac{E_{0}^{2}}{2 c M_{\mathrm{I}}} \operatorname{Re}\left[\left(\varepsilon_{\mathrm{I}}-\varepsilon_{\mathrm{II}}\left|M_{\mathrm{I}} / M_{\mathrm{II}}\right|^{4}\right) E_{r}^{(w)} E_{r}^{(w)^{*}}+\left(\varepsilon_{\mathrm{II}}-\varepsilon_{\mathrm{I}}\right)\right. \\
& \left.\times\left(E_{\theta}^{(w)} E_{\theta}^{(w)^{*}}+E_{\phi}^{(w)} E_{\phi}^{(w)^{*}}\right)\right]_{r=a} \mathbf{e}_{r},
\end{aligned}
$$

where $c$ is the speed of light. The stress is directed along the 


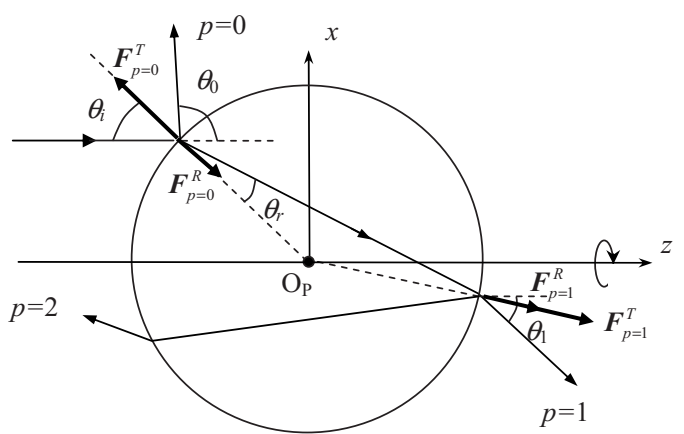

FIG. 2. Geometrical optics model of a sphere for the stress calculation. When a ray is incident on the sphere $(p=0)$, the reflection force $F_{p=0}^{R}(<0)$ and refraction force $F_{p=0}^{T}(>0)$ are in opposite directions. When the ray is transmitted through the sphere $(p=1)$, the reflection and refraction forces are in the same direction, namely, $F_{p=1}^{R}>0$ and $F_{p=1}^{T}>0$.

outward surface normal $\mathbf{e}_{r}$ when $\boldsymbol{f}>0$ and opposite to it when $f<0$.

The electric field $E_{0}$ at the center of the beam focal waist is related to the beam power $P$. When a Gaussian beam of fundamental mode is used, we have

$$
E_{0}^{2}=\frac{2 P}{\pi w_{0}^{2}\left(1+s^{2}+1.5 s^{4}\right)},
$$

where $w_{0}$ is the waist radius and $s=\lambda / 2 \pi w_{0}$ is the beam confinement parameter.

Note that for an absorbing sphere, in addition to the surface stress the force density is also distributed inside the sphere. Thus the net force in Minkowski form is equal to the surface integral of the stress over the particle surface plus the volume integral of the force density inside the particle, namely,

$$
\boldsymbol{F}=\boldsymbol{F}_{\text {surf }}+\boldsymbol{F}_{\mathrm{abs}}=\int \boldsymbol{f}_{\text {surf }} d S+\int \boldsymbol{f}_{\mathrm{abs}} d V,
$$

where the force density due to absorption is related to the Poynting vector inside the particle by

$$
\boldsymbol{f}_{\mathrm{abs}}=\frac{1}{2} k_{0} \operatorname{Im}\left(M_{\mathrm{II}}^{2}\right) \operatorname{Re}\left(\mathbf{E} \times \mathbf{H}^{*}\right) .
$$

\section{GEOMETRICAL OPTICS}

\section{A. Preliminaries}

To avoid the mathematical and numerical complexity of electromagnetic theory, GO is developed as an approximate method for stress prediction. In GO, the incident beam is decomposed into a collection of individual rays carrying a certain amount of momentum and propagating in different directions. Each time the ray is reflected or refracted into or out of the particle, a fraction of the incident momentum is imparted to the particle so that a reaction force is exerted. The final stress is obtained through an incoherent summation of all the force components acting on the same surface element. A sketch of ray interaction with a sphere is illustrated in Fig. 2.
Consider a Gaussian beam focused by a lens to a focal waist of half-width $w_{0}$. It propagates along the positive $z$ direction and the center of its focal waist has coordinates $\mathrm{O}_{\mathrm{B}}\left(0,0, z_{0}\right)$ in the reference frame of the particle. The electric amplitude of the beam is described by [19]

$$
E(x, y, z)=E_{0} \frac{w_{0}}{w} \exp \left(-\frac{x^{2}+y^{2}}{w^{2}}\right) \exp \left(i \varphi_{i}\right)
$$

where $E_{0}$ denotes the amplitude of the central ray of the beam, $\varphi_{i}$ is the phase term, and $w$ is the local waist radius along the beam axis given by $w=w_{0} \sqrt{1+\left[\left(z-z_{0}\right) \lambda / \pi w_{0}^{2}\right]^{2}}$.

The propagation direction of an arbitrary ray should be determined by taking the normal of the local phase plane at the surface area element where the ray intersects the particle [19]. However, for simplification, assume that all the incident rays are parallel and intersect the particle surface with various incidence angles $\theta_{i}\left(0 \leq \theta_{i} \leq \pi / 2\right)$.

\section{B. Momentum transfer}

The center of the sphere is assumed to be located on the beam axis. In this case, the plane of incidence at every interaction of a ray with the particle surface is identical, the TE and TM polarizations always stay separate, and the calculation can be greatly simplified. Assigning the order $p=0$ to the first interaction between a ray and the sphere, the differential area $d A_{0}$, evaluated by

$$
d A_{0}=a^{2} \sin \theta_{i}\left|d \theta_{i}\right| d \phi,
$$

receives the power $\Pi$ from the incident annular bundle of rays:

$$
\Pi=|E|^{2} \cos \theta_{i} d A_{0}=E_{0}^{2}\left(\frac{w_{0}}{w}\right)^{2} \exp \left(-\frac{2 r^{2}}{w^{2}}\right) \cos \theta_{i} d A_{0} .
$$

The rate of momentum of the incident annular bundle of rays, $\boldsymbol{M}_{0, i}$ (the second subscript $i$ denotes the incident rays), can be evaluated by the following expression when it propagates along the positive $z$ axis in a medium of refractive index $M_{\mathrm{I}}$ :

$$
\boldsymbol{M}_{0, i}=\frac{\Pi M_{\mathrm{I}}}{c} \hat{z}
$$

Taking advantage of the spherical symmetry of the particle, we consider ray tracing in the $\mathrm{O}_{\mathrm{P}}-x z$ plane. When a ray hits the surface of the particle, a fraction of its energy is reflected. The rate of momentum of the externally reflected ray bundle (denoted by the subscript $r$ ) is

$$
\boldsymbol{M}_{0, r}=\frac{\Pi M_{\mathrm{I}}}{c} r_{j}^{2}\left[\cos \left(\pi-2 \theta_{i}\right) \hat{z}+\sin \left(\pi-2 \theta_{i}\right) \hat{x}\right],
$$

where $r_{j}$ are intensity Fresnel coefficients, with $j$ being 1 for TE polarization (along the $x$ axis) and 2 for TM polarization (along the $y$ axis). The remainder of the incident energy is refracted into the sphere so that the refracted ray bundle (denoted by the subscript $t$ ) has the following momentum rate: 


$$
\boldsymbol{M}_{0, t}=\frac{\Pi M_{\mathrm{r}} M_{\mathrm{I}}}{c}\left(1-r_{j}^{2}\right)\left\{\cos \left[-\left(\theta_{i}-\theta_{r}\right)\right] \hat{z}+\sin \left[-\left(\theta_{i}-\theta_{r}\right)\right] \hat{x}\right\},
$$

where $\theta_{r}$ is the angle of refraction and $M_{\mathrm{r}}$ is the real part of refractive index of the particle relative to the medium, namely, $M_{\mathrm{r}}=\operatorname{Re}\left(M_{\mathrm{II}} / M_{\mathrm{I}}\right)$.

When a ray bundle encounters the boundary of the particle for the $(p+1)$ th time, the order $p$ is assigned. The momentum rate of the reflected ray bundle of $p$ th order is

$$
\begin{aligned}
\boldsymbol{M}_{p, r}= & \frac{\Pi M_{\mathrm{r}} M_{\mathrm{I}}}{c} \exp \left(-4 p k_{\mathrm{I}} a M_{\mathrm{i}} \cos \theta_{r}\right)\left(1-r_{j}^{2}\right) r_{j}^{2 p} \\
& \times\left\{\cos \left[-\left(\theta_{i}-\theta_{r}\right)-p\left(\pi-2 \theta_{r}\right)\right] \hat{z}\right. \\
& \left.+\sin \left[-\left(\theta_{i}-\theta_{r}\right)-p\left(\pi-2 \theta_{r}\right)\right] \hat{x}\right\} .
\end{aligned}
$$

The momentum rate of the refracted (or transmitted) ray bundle of $p$ th order is

$$
\begin{aligned}
\boldsymbol{M}_{p, \mathrm{t}}= & \frac{\Pi M_{\mathrm{I}}}{c} \exp \left(-4 p k_{\mathrm{I}} a M_{\mathrm{i}} \cos \theta_{r}\right)\left(1-r_{j}^{2}\right)^{2} r_{j}^{2(p-1)} \\
& \times\left\{\cos \left[-2\left(\theta_{i}-\theta_{r}\right)-(p-1)\left(\pi-2 \theta_{r}\right)\right] \hat{z}\right. \\
& \left.+\sin \left[-2\left(\theta_{i}-\theta_{r}\right)-(p-1)\left(\pi-2 \theta_{r}\right)\right] \hat{x}\right\} .
\end{aligned}
$$

In Eqs. (24) and (25), $M_{\mathrm{i}}$ is the imaginary part of relative refractive index, namely, $M_{\mathrm{i}}=\operatorname{Im}\left(M_{\mathrm{II}} / M_{\mathrm{I}}\right)$, the exponential term denotes the amplitude attenuation due to absorption, and the term $\left(1-r_{j}^{2}\right) r_{j}^{2(p-1)}$ represents the fraction of the en- ergy remaining in the ray after $(p-1)$ internal reflections.

The change in momentum rate must be balanced by a reaction force $\boldsymbol{F}_{p}$ :

$$
\boldsymbol{F}_{p}=\boldsymbol{f}_{p} d A_{p}=\boldsymbol{M}_{p, i}-\left(\boldsymbol{M}_{p, r}+\boldsymbol{M}_{p, t}\right),
$$

where $\boldsymbol{M}_{p, i}$ is given by Eq. (21) for $p=0$, by $M_{p-1, t} \exp$ $\left(-4 k_{\mathrm{I}} a M_{\mathrm{i}} \cos \theta_{i}\right)$ for $p=1, \quad$ and by $M_{p-1, r} \exp$ $\left(-4 k_{\mathrm{I}} a M_{\mathrm{i}} \cos \theta_{r}\right)$ for $p \geq 2$. The reaction force of order $p$ acts on a differential area $d A_{p}$ on the sphere surface,

$$
d A_{p}=a^{2} \sin \theta_{p}^{L}\left|d \theta_{p}^{L}\right| d \phi,
$$

parametrized by the angle $\theta_{p}^{L}$ that the location of the differential area makes with the positive $z$ axis. The angle $\theta_{p}^{L}$ characterizes the location of a ray's exit (or incident) point on the sphere surface. It is related to the total angular deviation $\theta_{p}^{\prime}$ of the emergent ray with respect to the $z$ axis by

$$
\theta_{p}^{L}=\left(\theta_{p}^{\prime}+\theta_{i}\right)-2 \pi k,
$$

where $k$ is an integer restricting $\theta_{p}^{L}$ to the angular range $[0,2 \pi]$ on the sphere surface and $\theta_{p}^{\prime}$ is evaluated by

$$
\theta_{p}^{\prime}=2 p \theta_{r}-2 \theta_{i}-(p-1) \pi,
$$

A superposition of different order stresses at the same place produces the final surface stress:

$$
\boldsymbol{f}_{\text {surf }}=\sum_{p=0} \boldsymbol{f}_{p},
$$

where

$$
f_{p}= \begin{cases}E_{0}^{2} \frac{M_{\mathrm{I}}}{c}\left(\frac{w_{0}}{w}\right)^{2} \exp \left(-\frac{2 r^{2}}{w^{2}}\right) \cos \theta_{i}(A \hat{z}+B \hat{x}), & p=0 \\ E_{0}^{2} \frac{M_{\mathrm{I}}}{c}\left(\frac{w_{0}}{w}\right)^{2} \exp \left(-\frac{2 r^{2}}{w^{2}}\right) \cos \theta_{i} \frac{d A_{0}}{d A_{p}} \exp \left(-4 p k_{\mathrm{I}} a M_{\mathrm{i}} \cos \theta_{r}\right)\left(1-r_{j}^{2}\right) r_{j}^{2(p-1)}(C \hat{z}+D \hat{x}), & p \geq 1,\end{cases}
$$

and

$$
A=1-r_{j}^{2} \cos \left(\pi-2 \theta_{i}\right)-M_{\mathrm{r}}\left(1-r_{j}^{2}\right) \cos \left[-\left(\theta_{i}-\theta_{r}\right)\right],
$$

$$
B=0-r_{j}^{2} \sin \left(\pi-2 \theta_{i}\right)-M_{\mathrm{r}}\left(1-r_{j}^{2}\right) \sin \left[-\left(\theta_{i}-\theta_{r}\right)\right],
$$

$$
\begin{aligned}
C= & M_{\mathrm{r}} \cos \left[-\left(\theta_{i}-\theta_{r}\right)-(p-1)\left(\pi-2 \theta_{r}\right)\right]-M_{\mathrm{r}} r_{j}^{2} \\
& \times \cos \left[-\left(\theta_{i}-\theta_{r}\right)-p\left(\pi-2 \theta_{r}\right)\right]-\left(1-r_{j}^{2}\right) \\
& \times \cos \left[-2\left(\theta_{i}-\theta_{r}\right)-(p-1)\left(\pi-2 \theta_{r}\right)\right],
\end{aligned}
$$

$$
\begin{aligned}
D= & M_{\mathrm{r}} \sin \left[-\left(\theta_{i}-\theta_{r}\right)-(p-1)\left(\pi-2 \theta_{r}\right)\right]-M_{\mathrm{r}} r_{j}^{2} \\
& \times \sin \left[-\left(\theta_{i}-\theta_{r}\right)-p\left(\pi-2 \theta_{r}\right)\right]-\left(1-r_{j}^{2}\right) \\
& \times \sin \left[-2\left(\theta_{i}-\theta_{r}\right)-(p-1)\left(\pi-2 \theta_{r}\right)\right],
\end{aligned}
$$

$$
\frac{d A_{0}}{d A_{p}}=\frac{\sin \theta_{i}}{\sin \theta_{p}^{L}}\left|\frac{d \theta_{p}^{L}}{d \theta_{i}}\right|=\frac{\sin \theta_{i}}{\sin \theta_{p}^{L}}\left|\frac{\sqrt{M_{\mathrm{r}}^{2}-\sin ^{2} \theta_{i}}}{\left(2 p \cos \theta_{i}-\sqrt{M_{\mathrm{r}}^{2}-\sin ^{2} \theta_{i}}\right)}\right| .
$$

Substituting the Fresnel coefficients into Eq. (31) and invoking the Snell-Descartes law of refraction, the angular component of the force is found to be zero and only the radial component remains.

As a special case, a compact form is available for the stress of the first two orders $p=0$ and 1 on a nonabsorbing sphere surface: 


$$
f_{p=0}=E_{0}^{2} \frac{2 M_{\mathrm{I}}}{c}\left(\frac{w_{0}}{w}\right)^{2} \exp \left(-\frac{2 r^{2}}{w^{2}}\right) \cos ^{2} \theta_{i} \begin{cases}\frac{M_{\mathrm{II}}^{2}-M_{\mathrm{I}}^{2}}{\left(M_{\mathrm{I}} \cos \theta_{i}+M_{\mathrm{II}} \cos \theta_{r}\right)^{2}} \hat{r}, & \mathrm{TE} \\ \frac{\left[M_{\mathrm{II}}^{2}-\left(M_{\mathrm{II}}^{2}-M_{\mathrm{I}}^{2}\right) \cos ^{2} \theta_{r}\right]\left(M_{\mathrm{II}}^{2}-M_{\mathrm{I}}^{2}\right)}{M_{\mathrm{I}}^{2}\left(M_{\mathrm{I}} \cos \theta_{r}+M_{\mathrm{II}} \cos \theta_{i}\right)^{2}} \hat{r}, & \mathrm{TM},\end{cases}
$$

and

$$
f_{p=1}=E_{0}^{2} \frac{2 M_{\mathrm{I}}}{c}\left(\frac{w_{0}}{w}\right)^{2} \exp \left(-\frac{2 r^{2}}{w^{2}}\right) \frac{d A_{0}}{d A_{p}} \cos ^{2} \theta_{r} \times\left\{\begin{array}{l}
\left(\frac{2 M_{\mathrm{II}} \cos \theta_{i}}{M_{\mathrm{I}} \cos \theta_{i}+M_{\mathrm{II}} \cos \theta_{r}}\right)^{2} \frac{M_{\mathrm{II}}^{2}-M_{\mathrm{I}}^{2}}{\left(M_{\mathrm{I}} \cos \theta_{i}+M_{\mathrm{II}} \cos \theta_{r}\right)^{2}} \hat{r}, \\
\left(\frac{2 M_{\mathrm{II}} \cos \theta_{i}}{M_{\mathrm{II}} \cos \theta_{i}+M_{\mathrm{I}} \cos \theta_{r}}\right)^{2} \frac{\left(M_{\mathrm{II}}^{2}-M_{\mathrm{I}}^{2}\right)\left[M_{\mathrm{I}}^{2}+\left(M_{\mathrm{II}}^{2}-M_{\mathrm{I}}^{2}\right) \cos ^{2} \theta_{i}\right]}{M_{\mathrm{II}}^{2}\left(M_{\mathrm{I}} \cos \theta_{r}+M_{\mathrm{II}} \cos \theta_{i}\right)^{2}} \hat{r},
\end{array}\right.
$$

respectively. These two orders provide the major contributions to the surface stress since in GO calculation more than $\sim 75 \%$ intensity of an incident ray is attenuated at these two orders.

\section{NUMERICAL IMPLEMENTATION}

Numerical procedures have been developed to implement the theory presented in Secs. II and III. For the evaluation of beam shape coefficients in GLMT, the fifth-order description of a Gaussian beam by Barton and Alexander [20] is used. All the numerical results presented in this section are for the laser power $P=1.0 \mathrm{~W}$. Therefore a multiplicative factor is needed for other beam powers.

As a validation of the GLMT stress prediction, the integral of the stress profile over the surface of a nonabsorbing sphere has been carried out and the results agree perfectly with the net force calculated from the analytical solution [10]. For an absorbing sphere, however, the optical force is not only distributed on its surface but also throughout its interior. In this case, Eq. (16) was verified. Since most calculation in this section concerns a nearly transparent droplet of radius $20 \mu \mathrm{m}$ and with the imaginary part of the refractive index as small as $10^{-7}$, the absorption force $\boldsymbol{F}_{\text {abs }}$ is negligible and deformation of the particle can be considered as resulting from the surface stress.

\section{A. Plane-wave incidence}

Let a water droplet of radius $a=20 \mu \mathrm{m}$ be illuminated by a linearly polarized beam (in the $x$ direction at the waist) produced by a cw Ti:sapphire laser $\left(\lambda_{0}=0.785 \mu \mathrm{m}\right)$. The droplet has the refractive index $M_{\mathrm{II}}=1.330+1.342 \times 10^{-7} i$ at the incident wavelength [21]. The beam is focused to a waist radius equal to ten times the particle radius $\left(w_{0}=10 a\right)$ so that it can be viewed as a plane wave with respect to the particle size. GLMT is used to obtain a rigorous picture of the stress. Its prediction of the stress profile in the plane of azimuthal angle $\phi=0^{\circ}$ is plotted in Cartesian and polar coordinates, respectively, in Figs. 3(a) and 3(b). (Due to the axial symmetry of the stress about the $z$ axis in the current case
$\left[f\left(\theta^{L}\right)=f\left(360^{\circ}-\theta^{L}\right)\right]$, the stress is plotted only for $0^{\circ} \leq \theta^{L}$ $\leq 180^{\circ}$ in Fig. 3(a).) As an approximate method, GO calculates the stress contributed by rays of order $p=0+1$ in these figures. Stresses of these two order rays do not superpose since the $p=0$ and 1 order rays contribute stress on the surfaces of the illuminated hemisphere $\left(90^{\circ} \leq \theta_{p=0}^{L} \leq 180^{\circ}\right)$ and shadowed hemisphere $\left(0^{\circ} \leq \theta_{p=1}^{L} \leq 90^{\circ}\right)$, respectively. To evaluate the error of $\mathrm{GO}$ in calculating the $p=0+1$ order stress, the result is compared in Fig. 4(a) to the prediction of the $p=0+1$ Debye series which can accurately access the stress of a given order. Perfect agreement of stresses contributed by $p=0$ order rays can be observed on the illuminated surface $\left(90^{\circ} \leq \theta^{L} \leq 180^{\circ}\right)$. On the shadowed surface, however, the $p=1$ stress calculated by Debye series and GO differ remarkably from each other within the angular interval $0^{\circ} \leq \theta^{L}<30^{\circ}$ because of the wave theory oscillatory structure.

To explore the reason for this deviation, we first exclude the influence of the diffracted wave (DW). An argument is provided in the Appendix showing that for a transparent sphere the diffracted wave is canceled by the incident wave (IW) on shadowed hemisphere. On the illuminated hemisphere, it has no essential contribution either. Further, the location of the exit point of the $p=1$ ray versus the incidence angle of the original rays is illustrated in Fig. 4(b). It can be observed that as the incident ray departs from $z$ axis (or as the incidence angle $\theta_{i}$ increases), the location of the exit point first increases to $\theta_{\mathrm{fc}}^{L}=21.258^{\circ}$ and then decreases to $\theta_{\text {edge }}^{L}=7.507^{\circ}$. The edge ray of grazing incidence causes a force at $\theta_{\mathrm{edge}}^{L}$, while $\theta_{\mathrm{fc}}^{L}$ implies a transition point from tworay region $\left(\theta_{\text {edge }}^{L}<\theta^{L}<\theta_{\mathrm{fc}}^{L}\right)$ to zero-ray region $\left(\theta^{L}>\theta_{\mathrm{fc}}^{L}\right)$, or a "fold caustic" (denoted by the subscript "fc") at which force density is extremely high due to the stationary turning of the ray's exit point on the sphere surface with respect to the incidence angle. The term "two-ray" means that there exist two different rays exiting (or incident on) the surface at the same point which is characterized by $\theta^{L}[22]$. Consequently, the two-ray interference causes a supernumerary structure for $\theta_{\text {edge }}^{L}<\theta^{L}<\theta_{\text {fc }}^{L}$. Mathematically, fold caustics in GO can be determined through letting $d \theta_{p}^{L} / d \theta_{i}=0$ so that 

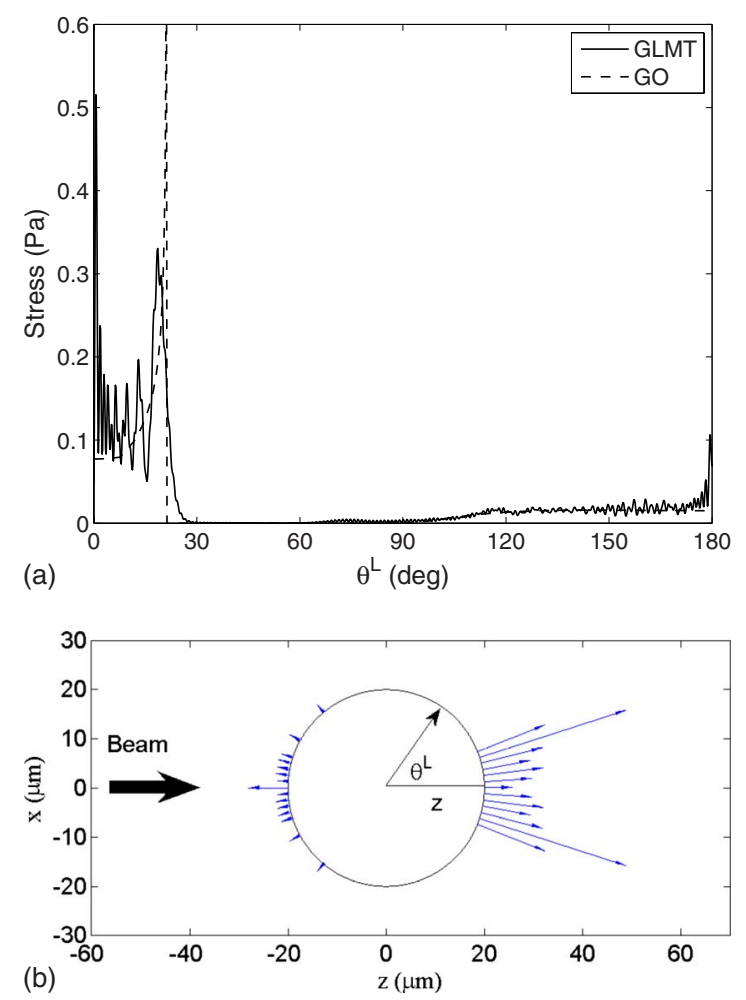

FIG. 3. (Color online) Stress profile on the surface of a spherical water droplet of radius $a=20 \mu \mathrm{m}$ and refractive index $M_{\mathrm{II}}=1.330$ $+1.342 \times 10^{-7} i$ and illuminated by a polarized (in the $x$ direction at the waist) Gaussian beam of wavelength $\lambda_{0}=0.785 \mu \mathrm{m}$ and waist radius equal to ten times the particle radius $\left(w_{0}=10 a=200 \mu \mathrm{m}\right)$. The droplet center coincides with that of the beam. The laser power is $P=1.0 \mathrm{~W}$. (a) Cartesian plot of the stress in the plane of the azimuthal angle $\phi=0^{\circ}$; (b) visualization of the GLMT stress plotted in (a).

$$
\begin{aligned}
\theta_{p, \mathrm{fc}}^{L}= & {\left[2 p \arcsin \left(\frac{1}{M_{\mathrm{r}}} \sqrt{\frac{4 p^{2}-M_{\mathrm{r}}^{2}}{4 p^{2}-1}}\right)-\arcsin \left(\sqrt{\frac{4 p^{2}-M_{\mathrm{r}}^{2}}{4 p^{2}-1}}\right)\right.} \\
& -(p-1) \pi]-2 \pi k
\end{aligned}
$$

An extremum ray of the incidence angle $\theta_{i}=59.585^{\circ}$ or impact parameter $x=0.862 a$ can be associated with the $p=1$ fold caustic point. The locus of these focal caustic points around the sphere surface forms the Descartes ring [23]. Comparison of Eq. (39) to the far-zone rainbow angle evaluated by letting $d \theta_{p}^{\prime} / d \theta_{i}=0$ shows that the incidence angle corresponding to the $p$ th-order fold caustic on the sphere surface is identical to the incidence angle of the $(2 p-1)$ th-order far-zone rainbow. The angular region $0^{\circ}$ $<\theta^{L}<\theta_{\text {edge }}^{L}$ is the one-ray region when viewed from GO which excludes the contribution of the edge rays missing striking the sphere. According to the wave theory, the partial waves corresponding to these edge rays produce electromagnetic surface waves. The surface wave rays travel along the circumference of the particle and interfere constructively or destructively with near-paraxial rays exiting the sphere at the same place so that the supernumerary structure continues to
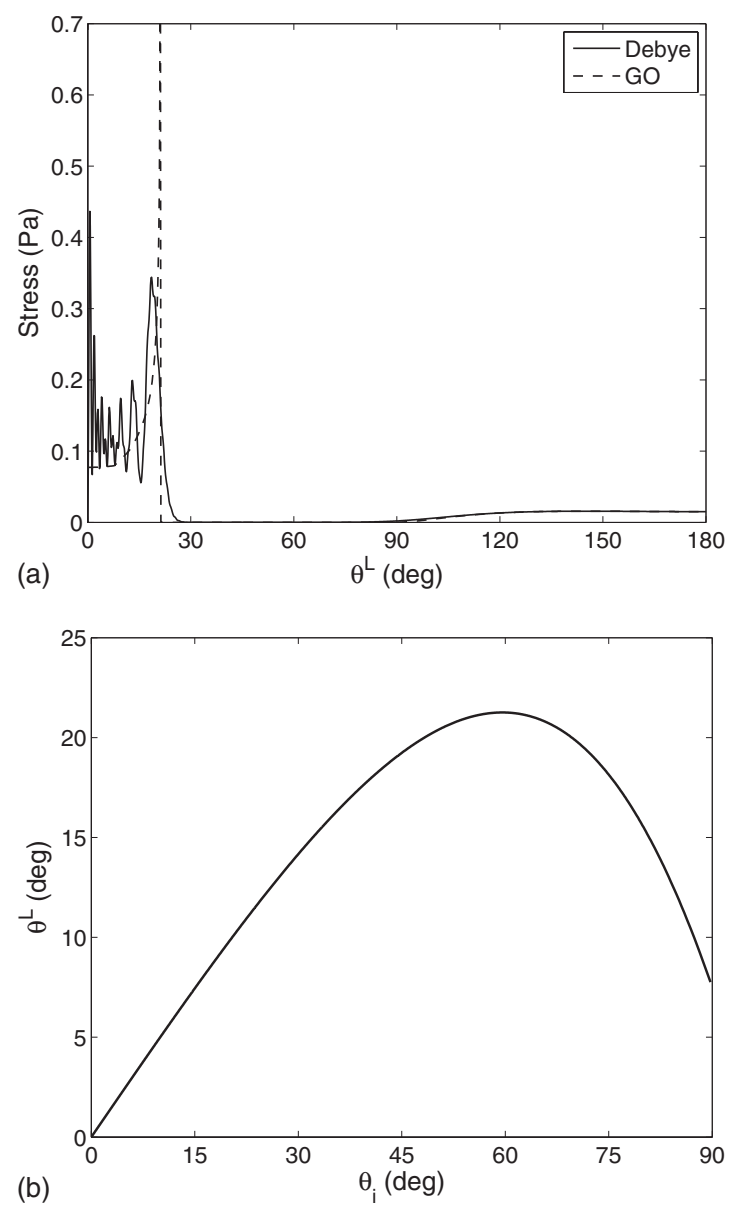

FIG. 4. (a) Comparison of the Debye series and the GO analysis of the stress of order $p=0+1$ in the plane of azimuthal angle $\phi$ $=0^{\circ}$. The parameters of the beam and the particle are the same as in Fig. 3. (b) Location of the exiting ray of order $p=1$ on the sphere surface versus the incidence angle. The parameters of the beam and the particle are the same as in Fig. 3.

exist. Using the method of Nussenzveig [24], the TE surface waves are estimated to travel an angular distance $\Delta \theta_{\mathrm{TE}}^{L}$ $=7.554^{\circ}$ on the surface of a sphere of size parameter $\alpha=k a$ $=160$ before their field amplitude drops to $1 / e$. The surface waves created at all points on the Descartes ring on the sphere surface converge together at $\theta^{L} \approx 0^{\circ}\left(\Delta \theta_{\mathrm{TE}}^{L}>\theta_{\text {edge }}^{L}\right)$ with constructive interference. However, compared to the remarkable amplification of the forward direction stress due to the convergence of the TE surface waves, the influence of TM surface waves on the stress in the plane $\phi=90^{\circ}$ is less (see Fig. 5). The TM surface waves travel an angular distance $\Delta \theta_{\mathrm{TM}}^{L}=7.091^{\circ}$, which is smaller than that of TE surface waves. They die off before converging at $\theta^{L} \approx 0^{\circ}\left(\Delta \theta_{\mathrm{TM}}^{L}\right.$ $<\theta_{\text {edge }}^{L}$ ). Lacking this convergence, TM surface waves only contribute an extension of the supernumerary structure into in the one-ray region $0^{\circ}<\theta^{L}<\theta_{\text {edge }}^{L}$. The interference amplification at $\theta^{L}=0^{\circ}$ is not as notable as in the plane $\phi=0^{\circ}$.

In addition to the supernumerary pattern, Figs. 3(a) and 5 show a jagged structure of the surface stress on the surface of the illuminated hemisphere $\left(\theta^{L} \geq 90^{\circ}\right)$, which is not observed in the Debye series calculation for order $p=0+1$ [Fig. 4(a)]. Since the $p=1$ order rays contributed the stress at $\theta^{L}<30^{\circ}$, 


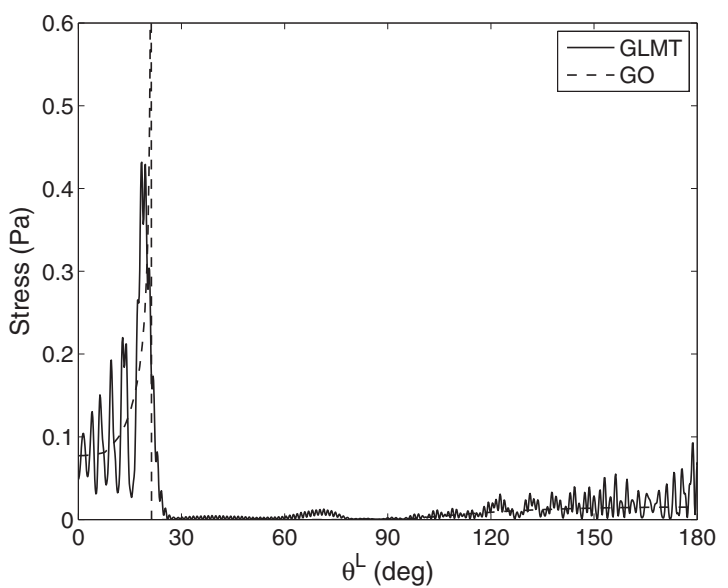

FIG. 5. Cartesian plot of the stress in the plane of azimuthal angle $\phi=90^{\circ}$. The parameters of the beam and the particle are the same as in Fig. 3.

the jagged structure is ascribed to the interference of the specularly reflected rays $(p=0)$ with higher-order rays. Stress components obtained by Debye series (to be clearly demonstrated in the upcoming Fig. 7) imply that the incident rays of the orders $p=2-4$ and 7 still contain considerable magnitude so as to interfere with the $p=0$ order rays and contribute the stress at the angles $\theta^{L} \geq 90^{\circ}$. A comparison of the stress contributed by $p=0+2+3+4+7$ to that of GLMT in the plane $\phi=0^{\circ}$ is made in Fig. 6, and shows good agreement.

Due to its exclusion of interference effects, the accuracy of the GO method cannot be improved by simply adding the contribution of higher-order rays $(p \geq 2)$. However, the main characteristics of the stress of separate orders can be physically understood by GO. For example, the fold caustics and axial caustic points can be well predicted by GO. The formation and effect of fold caustic point were analyzed above. In addition, an axial caustic (denoted by the subscript "ac") on the sphere surface is produced when rays in all planes of incidence exit the sphere surface from the point $z=a$ or $z=-a$, producing a stress maximum at $\theta^{L} \approx 0^{\circ}$ or $\theta^{L} \approx 180^{\circ}$.

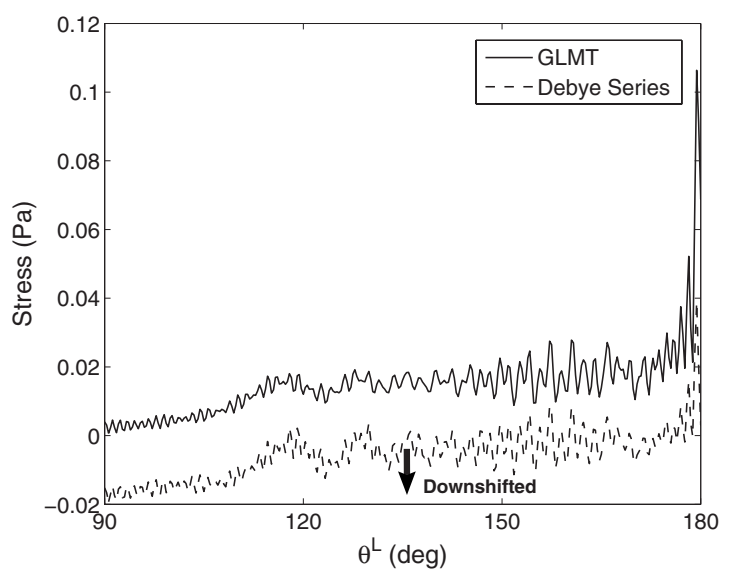

FIG. 6. Comparison of the Debye series calculation of the stress of order $p=0+2+3+4+7$ to the result of GLMT in the plane of the azimuthal angle $\phi=0^{\circ}$. (The Debye series curve is downward shifted by a factor of 0.02 for clarity.) The parameters of the beam and the particle are the same as in Fig. 3 .

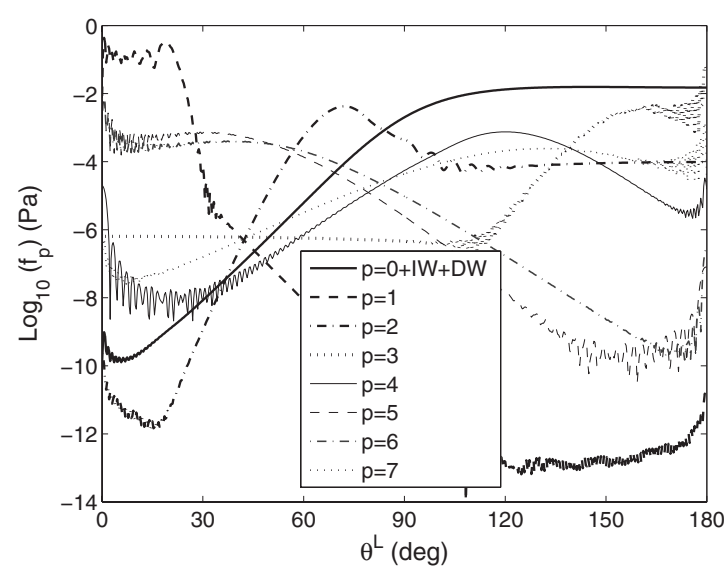

FIG. 7. Debye series analysis of the stress components $\left(f_{p}\right)$ of orders $p=0-7$ in the plane of the azimuthal angle $\phi=0^{\circ}$. (The IW and DW are included in the calculation of the stress of order $p=0$ and $f_{p}$ is plotted on a logarithmic scale). The parameters of the beam and the particle are the same as in Fig. 3.

To distinguish the contribution of different order caustics, the Debye series was used to evaluate the stress components contributed by the rays of orders $p=0-7$. The results are plotted on a logarithmic scale in Fig. 7. Note that the IW and DW are included in the calculation of $p=0$ order stress. The location of the exit points of the rays on sphere surface predicted by GO at these orders is given in Fig. 8(a). Each stationary turning point of $\theta_{p}^{L}$ with respect to the incidence angle $\theta_{i}$ is recognized as a fold caustic point. [Note that in Fig. 8(a) $\theta_{p}^{L}$ is calculated by $\theta_{p}^{L}=\theta_{p}^{\prime}+\theta_{i}$, without restriction to the angular interval $\left[0^{\circ}, 360^{\circ}\right]$.] The angle $\theta_{p}^{L}$ equal to $180^{\circ} k$ ( $k$ is an integer) is the location of an axial caustic point. Evaluated by GO, the maxima in the stress profile caused by fold caustic points should be located at $\theta_{p, \mathrm{GO}}^{L}=21.258^{\circ}$, $68.578^{\circ}, 153.255^{\circ}, 123.183^{\circ}, 40.055^{\circ}, 42.860^{\circ}$, and $125.654^{\circ}$ for $p=1-7$, respectively, which are identifiable in Fig. 7. The maxima correspond to the rays of initial incidence angles $\theta_{i}=59.585^{\circ}, 76.914^{\circ}, 81.476^{\circ}, 83.657^{\circ}$, $84.944^{\circ}, 85.795^{\circ}$, and $86.400^{\circ}$, respectively. As indicated by their flux fractions in Fig. 8(b), the incident rays of high orders corresponding to these incidence angles still carry considerable flux to contribute to the stress. As to the axial caustic points predicted by GO for different orders, the stress maxima induced by them can also be identified in the Debye stress profile at $\theta^{L} \approx 0^{\circ}$ or $\theta^{L} \approx 180^{\circ}$. Figure $8(\mathrm{~b})$ shows that the incident rays of a high order (e.g., $p \geq 5$ ) can have axial caustic points at both $\theta^{L} \approx 0^{\circ}$ and $\theta^{L} \approx 180^{\circ}$.

Next, we explore the role of absorption of the particle on the stress. The imaginary part of the particle refractive index is raised from $1.342 \times 10^{-7}$ to 15 . As indicated by Fig. 9, the stress distribution on the illuminated surface of the hemisphere becomes negative and little stress can be observed on the surface of the shadowed hemisphere. Since we define positive stress to be along the outwardly directed surface normal, the negative portion of Fig. 9 means an inwardly directed stress. At $\theta^{L}=180^{\circ}$, the negative stress pushes the particle along the beam propagation direction. To qualitatively analyze the negative stress, the radiation force is decomposed into two parts: one is from reflection and the other 

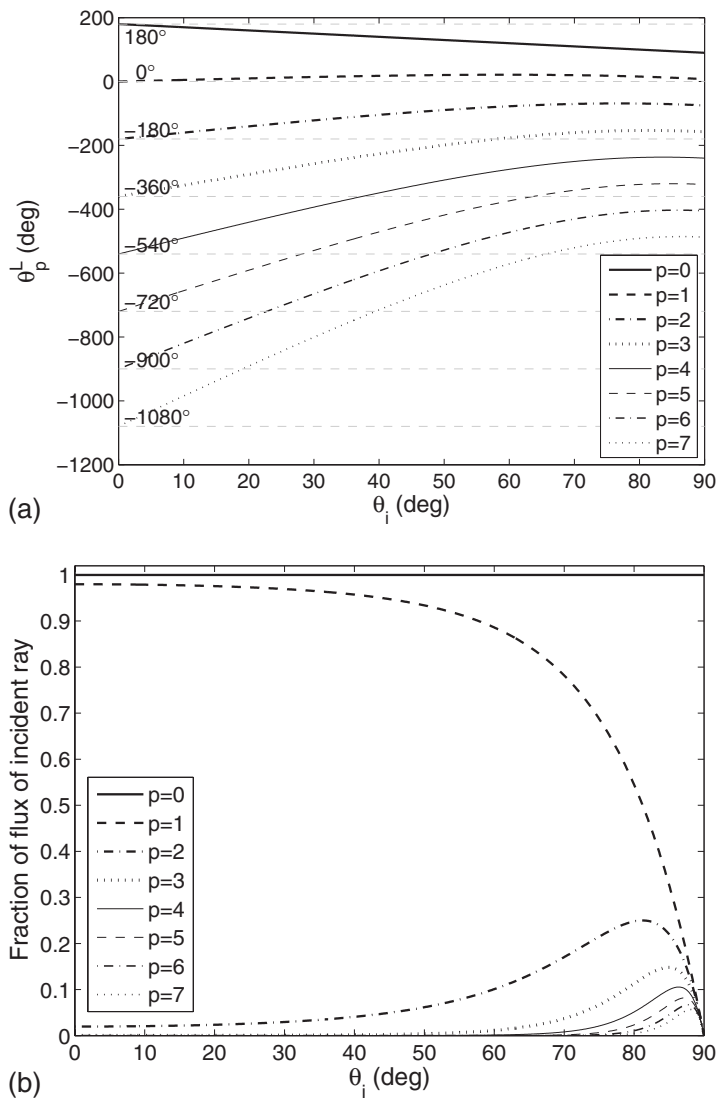

FIG. 8. GO evaluation of the exit ray's location of orders $p$ $=0-7$ on the sphere surface and the flux contained in the incident ray at these orders. The parameters of the beam and the particle are the same as in Fig. 3. (a) Location of the exiting ray versus the incidence angle (here $\theta_{p}^{L}$ is calculated by $\theta_{p}^{L}=\theta_{p}^{\prime}+\theta_{i}$, without restriction to the angular interval $\left.\left[0^{\circ}, 360^{\circ}\right]\right)$. (b) Fraction of the flux contained in the incident ray in plane of the azimuthal angle $\phi$ $=0^{\circ}$.

is from refraction. Their directions are opposite to each other for order $p=0$ when the ray is first refracted from the less optically dense medium (air) into the more optically dense medium (droplet): the reflection force $\boldsymbol{F}_{p=0}^{R}$ is directed toward the particle center, while the refraction force $\boldsymbol{F}_{p=0}^{T}$ is directed away from it. But at higher orders $(p \geq 1)$, when the ray is refracted from more optically dense medium (droplet) into the less optically dense medium (air), both the reflection force $\boldsymbol{F}_{p \geq 1}^{R}$ and the refraction force $\boldsymbol{F}_{p \geq 1}^{T}$ are outwardly directed (see Appendix A of Ref. [25] for a derivation and Fig. 2 for an illustration). For a nearly nonabsorbing sphere, most of the energy enters and leaves the particle via refraction at orders $p=0$ and 1 , respectively. The stress in both the forward and backward angular ranges is dominated by the positive refraction force. However, for an absorbing sphere, with an increase in the imaginary part of the refractive index, external reflection, which induces a negative force, becomes more significant. Moreover, more of the energy refracted into the particle is absorbed along the path through it, so less energy exits at the order $p=1$ and the decreased force amplitude is observed on the surface of the shadowed hemisphere. In the extreme case of $M_{\mathrm{II}}=1.330+15 i$, the particle nearly totally reflects the incident rays. Therefore the stress profile

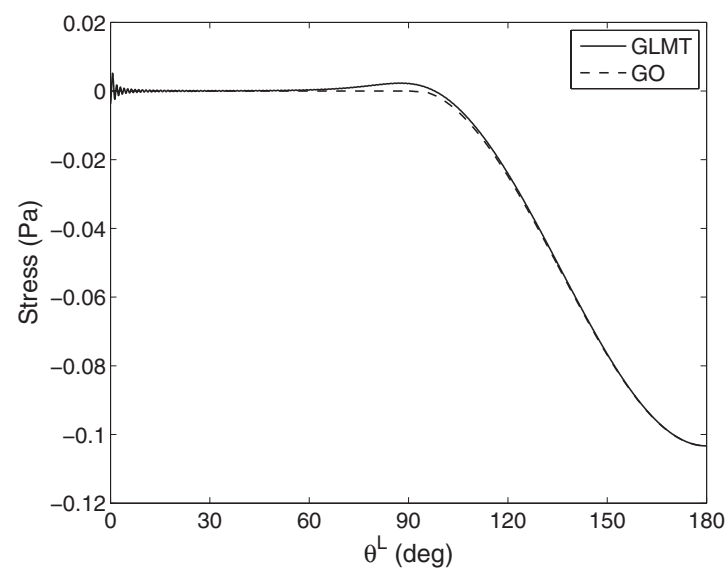

FIG. 9. Stress profile on the surface of a droplet. The parameters of the beam and the particle are the same as in Fig. 3 except that the refractive index of the particle is $M_{\mathrm{II}}=1.330+15 i$. The stress is in the plane of the azimuthal angle $\phi=0^{\circ}$.

is dominated by the negative reflection force of order $p=0$ and the stress on the shadowed surface is very weak. In addition, we show in the Appendix that the diffracted wave has no essential contribution to the stress on an impenetrable sphere surface.

\section{B. Gaussian-beam incidence}

\section{On-axis incidence}

To study the stress on the surface of a sphere located on the beam axis, the beam is focused to a waist radius slightly less than the particle radius $\left(w_{0}=0.8 a\right)$. In this case, the light flux contained in the edge rays that miss striking the sphere decreases and the interference of the surface wave with the directly transmitted rays of order $p=1$ in the near-forward directions is less pronounced. The two-ray interference is reduced as well. As a result, better agreement between the GO and GLMT results is observed in Fig. 10. Moreover, in

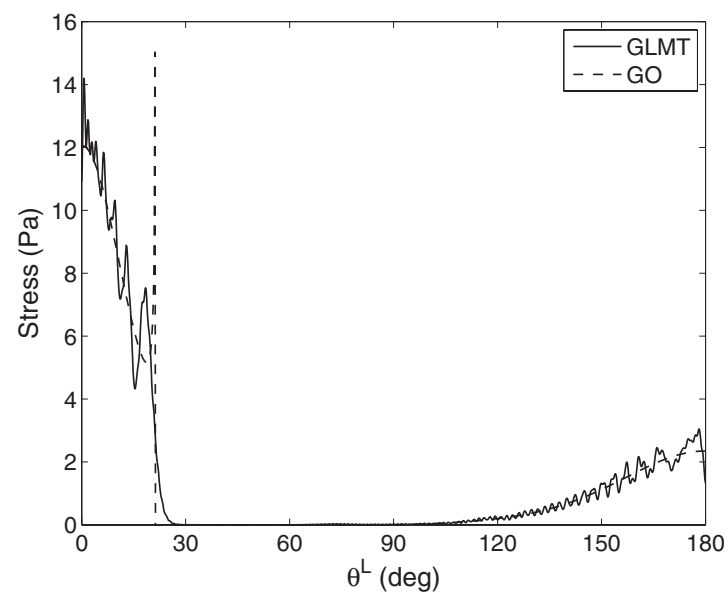

FIG. 10. Stress profile on the surface of a droplet. The parameters of the beam and the droplet are the same as in Fig. 3 except that waist radius is decreased to $w_{0}=0.8 a$. The stress is in the plane of the azimuthal angle $\phi=0^{\circ}$. 


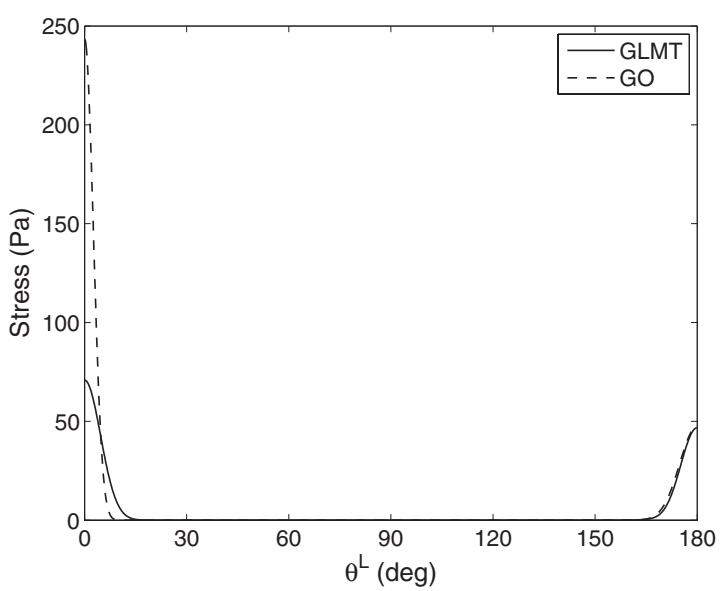

FIG. 11. Stress profile on the surface of a droplet. The parameters of the beam and the droplet are the same as in Fig. 3 except that waist radius is further decreased to $w_{0}=2 \lambda_{0} \approx 0.08 a$. The stress is in the plane of azimuthal angle $\phi=0^{\circ}$.

accordance with the nonuniformity of the incident-beam intensity, the stress on the shadowed hemisphere has a general decrease from the forward direction to larger angles.

Further, when the beam is focused to two times the wavelength $\left(w_{0}=2 \lambda_{0} \approx 0.08 a\right)$, in comparison to Figs. 3(a) and 10, the results plotted in Fig. 11 show an increase in stress in the forward and backward directions due to the fact that more momentum is carried by the incident rays and conveyed to the particle when the beam becomes more focused, while the laser power remains unchanged. Also in this case, the deviation of GO from GLMT is significant at the angles $\theta^{L}$ $<30^{\circ}$. One reason is that the description of a paraxial Gaussian beam of $\mathrm{TEM}_{00}$ mode by Eq. (18) and used by GO is actually the zeroth-order approximation of the realistic Gaussian beam described by an infinite series of contributions which rigorously satisfies Maxwell's equations [26]. In other words, Eq. (18) is valid only for a loosely focused beam with confinement parameter $s \ll 1$. Another reason is that the trajectories of the rays outside the particle are assumed to be linear and parallel to the beam axis. This assumption does not hold for a highly focused beam which exhibits considerable convergence or divergence. Our calculation shows that the inexact evaluation of the deflection angle can cause great deviation of the GO stress from the GLMT stress on the shadowed surface. Moreover, the GLMT stress profile in Fig. 11 does not show the supernumeraries any more, due to fact that the stress is dominated by the contribution of near-paraxial rays, and the momentum carried by the rays with impact parameter $x>0.862 a$, as well as those by the edge rays missing striking the sphere, is insufficient to cause either two-ray interference or one-ray and surface wave interference.

Next we explore the influence of the real part of the refractive index on the stress profile. An "optical stretcher," which is essentially a double beam trap employing two coaxial counterpropagating Gaussian beams to exert stress on a soft particle, is simulated. We calculate the stress exerted on the particle by one of the optical stretcher beams, which has the wavelength $\lambda_{0}=0.785 \mu \mathrm{m}$ and waist radius $w_{0}$
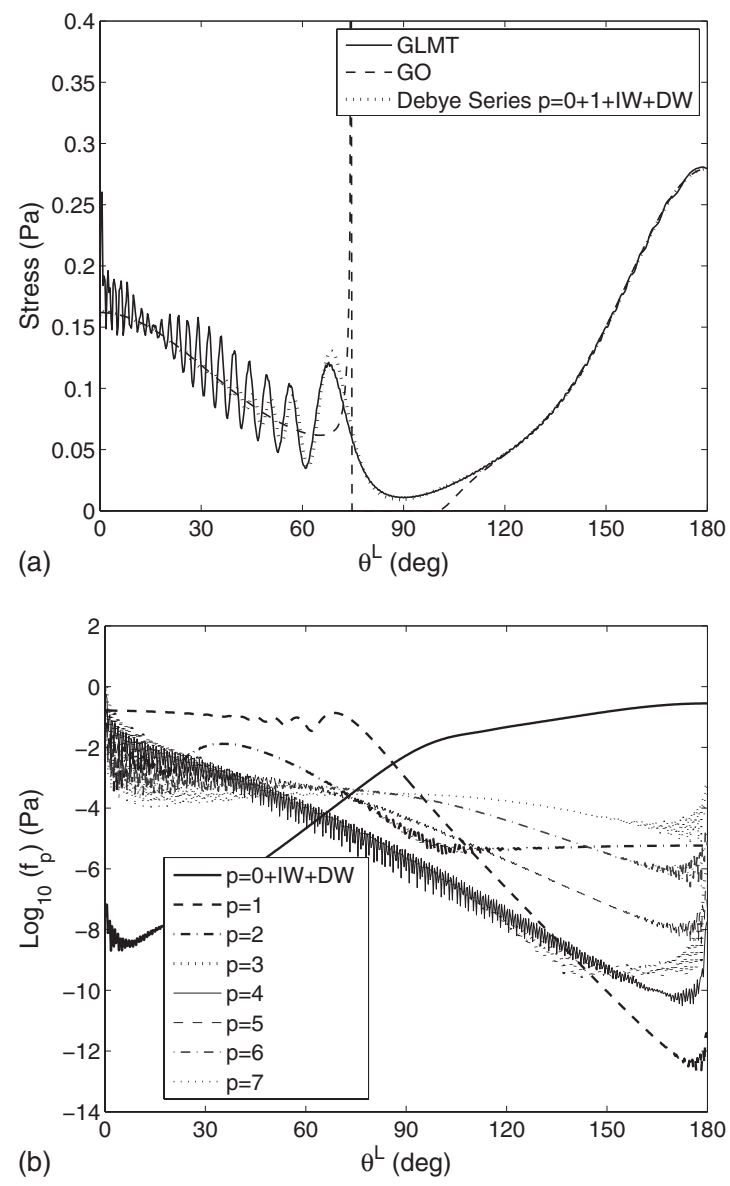

FIG. 12. Stress profile on the surface of a RBC of refractive index $M_{\mathrm{II}}=1.38+1.0 \times 10^{-5} i$ embedded in a buffer solution with osmolarity adjusted to $\sim 130 \mathrm{mOsm}$ so that its refractive index is $M_{\mathrm{I}}=1.335$. The beam has the wavelength $\lambda_{0}=0.785 \mu \mathrm{m}$ and is focused to the waist radius of $w_{0}=1.0 \mu \mathrm{m}$. The $\mathrm{RBC}$ has the radius $a=20.0 \mu \mathrm{m}$ and is trapped where the local waist radius is $10 \%$ larger than that of the particle $\left(w=1.10 a\right.$ and $\left.z_{0}<0\right)$. The stress is plotted in the plane of the azimuthal angle $\phi=0^{\circ}$. (a) Comparison between the stresses predicted by GLMT, GO, and the Debye series for $p=0+1$. (b) Debye series analysis of stress components $\left(f_{p}\right)$ of orders $p=0-7$ in the plane of the azimuthal angle $\phi=0^{\circ}\left(f_{p}\right.$ is plotted on a logarithmic scale).

$=1.0 \mu \mathrm{m}$. A red blood cell $(\mathrm{RBC})$ of refractive index $M_{\mathrm{II}}$ $=1.38+1.0 \times 10^{-5} i[27]$ is embedded in a buffer solution with osmolarity adjusted to $\sim 130 \mathrm{mOsm}$ so that its refractive index is $M_{\mathrm{I}}=1.335$ [2]. The $\mathrm{RBC}$ is trapped where the local waist radius is $10 \%$ larger than that of the particle $(w$ $=1.10 \mathrm{a}$ and $\left.z_{0}<0\right)$. To clearly demonstrate the physical characteristics, we assume the radius of the RBC to be as large as $a=20 \mu \mathrm{m}$ though it is typically less than $\sim 5 \mu \mathrm{m}$. Moreover, the thickness of the membrane is neglected here. Its influence on the stress profile will be analyzed in another paper.

Since the beam is highly focused and the particle is located in the divergent part of the beam, the incident rays can no longer be assumed parallel to the beam axis in GO. An inclination angle $\beta$ with respect to the $z$ axis has to be considered. In this case, the momentum carried by an incident ray becomes 


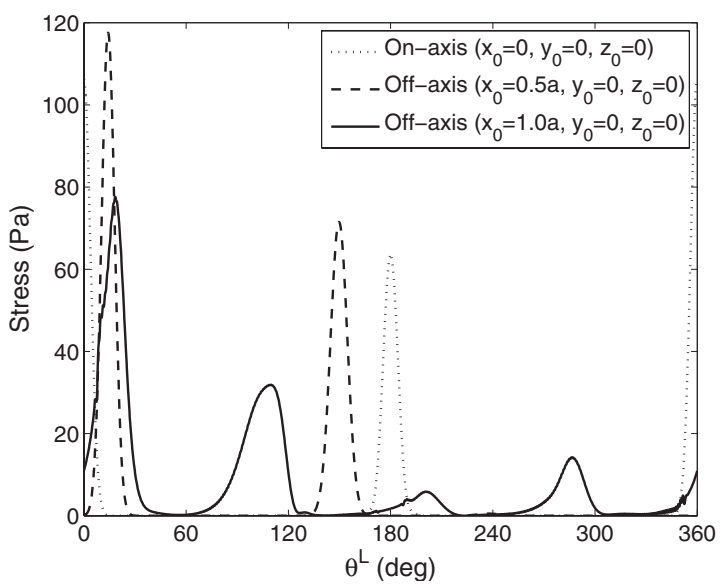

FIG. 13. Stress profile on the surface of a droplet of radius $a$ $=20 \mu \mathrm{m}$ and refractive index $M_{\mathrm{II}}=1.330+1.342 \times 10^{-7} i$ and illuminated by a polarized (in the $x$ direction at the waist) focused Gaussian beam of wavelength $\lambda_{0}=0.785 \mu \mathrm{m}$ and waist radius $w_{0}$ $=2 \mu \mathrm{m}$. The stress is plotted in the plane of the azimuthal angle $\phi=0^{\circ}$. The beam has its center $\mathrm{O}_{\mathrm{B}}\left(x_{0}, y_{0}, z_{0}\right)$ located at $(0,0,0)$, $(0.5 a, 0,0)$, and $(1.0 a, 0,0)$, respectively.

$$
\boldsymbol{M}_{0, i}=\frac{\Pi M_{\mathrm{I}}}{c}(\cos \beta \hat{z}+\sin \beta \hat{r}) .
$$

Likewise, $\beta$ has to be added in Eqs. (19), (22)-(25), (29), and (32) $-(36)$. Note that $\beta$ and $\theta_{i}$ are evaluated from the phase term $\varphi_{i}[19]$.

After this improvement, comparison of the GO- and GLMT-predicted stresses in the plane of the azimuthal angle $\phi=0^{\circ}$ is given in Fig. 12(a). Plotted in the same figure is the Debye series analysis of the stress for order $p=0+1$ (together with the IW and DW). Contrary to the droplet case, the interference structure of the stress on the illuminated hemisphere disappears as the refractive index of the particle approaches that of the medium $\left(M_{\mathrm{II}} / M_{\mathrm{I}} \sim 1.034\right)$, indicating that on the illuminated surface of the sphere $\left(\theta^{L}>90^{\circ}\right)$ most of the momentum transfer occurs as the ray is first refracted into the particle $(p=0)$. The Debye series analysis for $p=0$ +1 (black dotted line) displays the supernumerary structure at the angles close to the fold caustic point $\theta_{\mathrm{GO}}^{L}=74.5^{\circ}$. As discussed in Sec. IV A, the supernumerary structure is caused by the two-ray interference and extends to smaller angles with decreasing amplitude by the interference of one ray and the surface wave of order $p=1$. However, GLMT indicates a strengthened interference structure at the angles $\theta^{L}<60^{\circ}$. Analyzed by Debye series for stress orders $p$ $=0-7$ [see Fig. 12(b)], the interference at the angles $30^{\circ}$ $<\theta^{L}<60^{\circ}$ is found to be caused by the $p=1$ and $p=2$ interference. For $0^{\circ}<\theta^{L}<15^{\circ}$, interference of the $p=1$ ray with higher orders $p \geq 2$ causes the jagged structure.

\section{Off-axis incidence}

The influence of the beam location on the stress profile is studied in this subsection. We let a linearly polarized (in the $x$ direction at the waist) Gaussian beam with waist radius $w_{0}=2 \mu \mathrm{m}$ illuminate a droplet of radius $a=20 \mu \mathrm{m}$. Three different cases, namely, on-axis, off-axis, and edge inci-
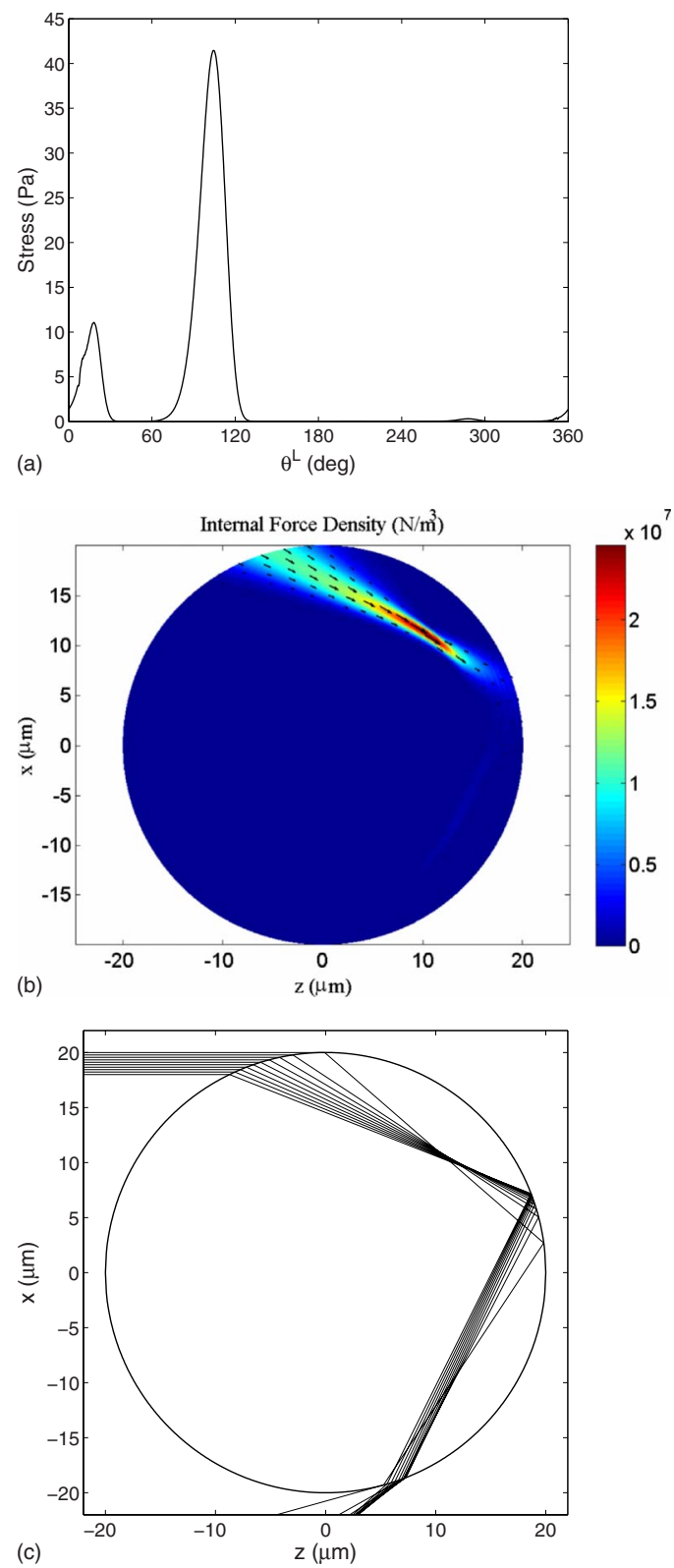

FIG. 14. (Color online) Stress distribution on the surface and force density in the interior of a droplet of radius $a=20 \mu \mathrm{m}$ and refractive index $M_{\mathrm{II}}=1.330+5.0 \times 10^{-3} i$ and illuminated at the edge $\left(x_{0}=a\right)$ by a polarized (in the $x$ direction at the waist) Gaussian beam of wavelength $\lambda_{0}=0.785 \mu \mathrm{m}$ and waist radius $w_{0}=2 \mu \mathrm{m}$. The stress is plotted in the plane of the azimuthal angle $\phi=0^{\circ}$. (a) Stress on the surface; (b) force density inside the sphere; (c) internal caustic structure in the plane $\mathrm{O}_{\mathrm{P}}-x z$ for a droplet of refractive index $M_{\mathrm{II}}=1.330$. Ten equidistant rays within the interval $a-w_{0} \leq x \leq a$ are incident on the droplet.

dences, are studied by letting the beam center $\mathrm{O}_{\mathrm{B}}$ be located at $(0,0,0),(0.5 a, 0,0)$, and $(1.0 a, 0,0)$, respectively, in the reference frame of the particle. The stress profiles in the azimuthal plane $\phi=0^{\circ}$ for these situations are predicted by GLMT and plotted in Fig. 13. It can be observed that with the beam moving from the on-axis incidence to off-axis incidence $\left(x_{0}=0.5 a\right)$, the first maximum moves from $\theta_{\mathrm{GLMT}}^{L}$ $=0^{\circ}$ to $14.2^{\circ}$. In the case of $x_{0}=0.5 a$, GO predicts that the 
incident ray carrying the maximum intensity has the incidence angle $\theta_{i, \mathrm{GO}}=30^{\circ}$ and contributes the maximum force at $\theta_{\mathrm{GO}}^{L}=150.0^{\circ}$ and $14.0^{\circ}$ for the orders $p=0$ and 1 , respectively. These angles agree with the GLMT-predicted maximum location at $\theta_{\mathrm{GLMT}}^{L}=149.7^{\circ}$ and $14.2^{\circ}$, respectively. When the beam center moves to the edge of the particle $\left(x_{0}=1.0 a\right)$, the $p=0$ stress maximum moves to $\theta^{L}=109.7^{\circ}$ and the first-order stress maximum $(p=1)$ moves to $\theta^{L}$ $=18.4^{\circ}$. A positive stress is produced at the angle $\theta^{L}=90^{\circ}$, which disagrees with the prediction of GO. In GO, the incident ray with maximum intensity has a grazing incidence angle and should be entirely reflected by the sphere so that a negative reflection force appears in the vicinity of $\theta^{L}=90^{\circ}$. However, in wave optics surface waves created by rays of near-grazing incidence [28] are refracted inside the sphere so that a positive refraction force is created, which overcomes the negative reflection force. Through ray tracing, it is found that the ray of incidence angle $\theta_{i, \mathrm{GO}}=70.3^{\circ}$ contributes the stress maximum at $\theta_{\mathrm{GO}}^{L}=109.7^{\circ}(p=0)$. Such an incident ray exits the sphere at $\theta_{\mathrm{GO}}^{L}=19.8^{\circ}(p=1)$, which is slightly larger than the GLMT prediction $\left(\theta_{\mathrm{GLMT}}^{L}=18.4^{\circ}\right)$. It further contributes the stress maxima in the directions $\theta_{\mathrm{GO}}^{L}=289.9^{\circ}(p=2)$ and $\theta_{\mathrm{GO}}^{L}=200.1^{\circ}(p=3)$, which are close to the GLMTpredicted locations of the two maxima at $\theta_{\mathrm{GLMT}}^{L}=287.0^{\circ}$ and $201.4^{\circ}$, respectively.

To study the force density distribution inside an absorbing particle, we let the beam illuminate the edge $\left(x_{0}=1.0 a\right)$ of a droplet of refractive index $M_{\mathrm{II}}=1.330+5.0 \times 10^{-3} i$. Compared to the case of a nearly nonabsorbing sphere (solid line in Fig. 13), the first maximum at $\theta^{L} \approx 18^{\circ}$ contributed by the stress of order $p=1$ on the shadowed surface is reduced [Fig. $14(\mathrm{a})]$ and the second and the third maxima at $\theta^{L} \approx 200^{\circ}$ and $287^{\circ}$ contributed by the rays of orders $p=3$ and 2 almost completely damp out. The absorption-induced force density distribution inside the droplet is illustrated in Fig. 14(b). Viewing the direction of the force lines, the laser-induced microflow of fluid inside an absorbing droplet can be imagined (in addition to the convection-induced flow caused by the thermal effect of the laser beam). Moreover, the high force density is located in the vicinity of $(z=10.5 \mu \mathrm{m}, x$ $=11 \mu \mathrm{m})$, which is near the point $(z=9.9 \mu \mathrm{m}, x$ $=11.3 \mu \mathrm{m})$, where the $p=1$ internal caustic of the grazing incidence ray is formed [29]. Viewed from GO, an internal caustic point is defined to be the intersection point of two adjacent rays propagating inside the sphere. The $p=1$ order ray theory caustic structure for a droplet of $M_{\mathrm{II}}=1.330$ is illustrated in Fig. 14(c). Ten equidistant rays within the interval $a-w_{0} \leq x \leq a$ were employed in the ray tracing. The GLMT prediction of Fig. 14(b) agrees well with the results of GO in Fig. 14(c). Increasing the refractive index to $M_{\mathrm{II}}$ $=1.330+1.0 \times 10^{-2} i$ leads to a further decrease in the first stress maximum [see Fig. 15(a)] and the high force density moves upstream from the internal caustic predicted by ray theory [see Fig. 15(b)]. Further increasing the absorption of the sphere by 1 order of magnitude $\left(M_{\mathrm{II}}=1.330+1.0\right.$ $\times 10^{-1} i$ ) leads to the observation (not shown in the paper) that the force damps in the skin layer of the sphere due to the rapid intensity decay via the exponential attenuation law after the rays are refracted into the particle.
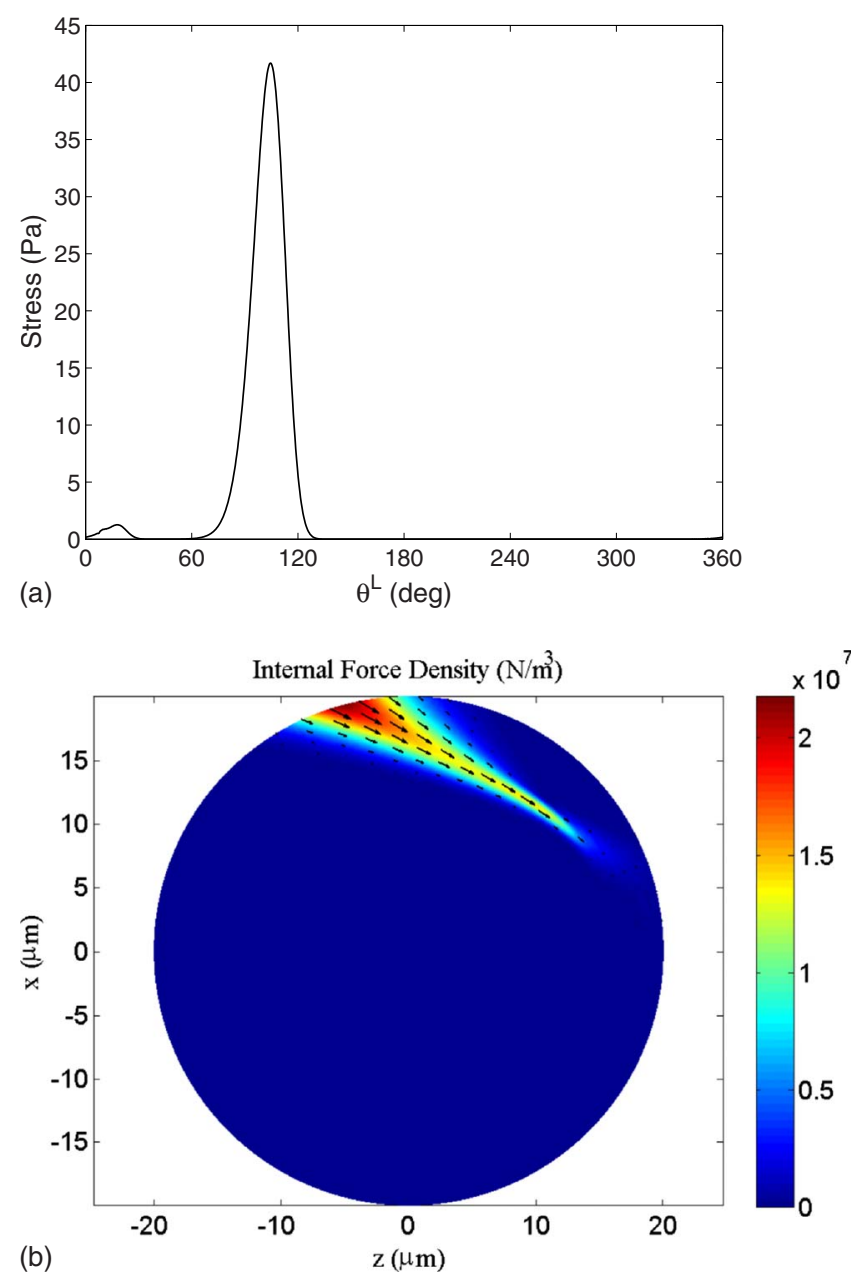

FIG. 15. (Color online) Stress distribution on the surface and force density in the interior of a droplet. The beam is as same as in Fig. 14, while the droplet has an increased imaginary part to its refractive index $M_{\mathrm{II}}=1.330+1.0 \times 10^{-2} i$. (a) Stress on the surface; (b) force density inside the sphere.

\section{CONCLUSION}

By using generalized Lorenz-Mie theory, geometrical optics, and the Debye series, the optical stress on a sphere surface is analyzed. It is found that the ratio of beam width to particle size and the refractive index of the particle have an important influence on the stress distribution. The inaccuracy of GO is not caused by its exclusion of the diffracted wave, but is due to the exclusion of surface waves and interference between rays when they superpose with each other in creating the stress on the sphere surface. For plane-wave incidence, two-ray interference of order $p=1$ causes the supernumerary structure on the surface of the shadowed hemisphere. The supernumerary structure is extended by the constructive or destructive interference between the surface wave rays and the near-paraxial rays exiting the sphere at the same place. Despite its inaccuracy, geometrical optics can be used for physically interpreting the characteristics of stress predicted by GLMT, e.g., the fold and axial caustic effects. GO agrees well with GLMT in predicting the force on the surface of a highly absorbing sphere which totally reflects 
the rays. However, inside an absorbing sphere the momentum transfer accompanies the propagation of the ray so that an internal force distribution is produced.

In response to the optical stress on the surface of a nonabsorbing soft spherical particle, the shape of the particle will distort, the optical stress on the surface will then change, and the shape will further distort until equilibrium is finally reached or the particle breaks. In the initial period of the deformation, the shape of the particle might be approximated by spheroid. Therefore as the next step, we aim to examine the matter-radiation interaction through calculating the optical profile for oblate and prolate spheroids of various eccentricities and orientations with respect to the incident-beam direction. These results will be published separately.

\section{ACKNOWLEDGMENTS}

This research was supported by Alexander von Humboldt Foundation (Grant No. CHN/1127072). In addition, F.X. is grateful to Dr. J. Guck for some constructive suggestions during his visit to Cavendish Laboratory of University of Cambridge and Dr. K. F. Ren for some beneficial discussions when he was in the CORIA at the University of Rouen.

\section{APPENDIX: ELECTROMAGNETIC FIELD DISTRIBUTION ON THE SURFACE OF A SPHERE ILLUMINATED BY GAUSSIAN BEAM}

\section{Preliminaries}

Consider a linearly polarized electromagnetic Gaussian beam of field strength $E_{0}$, polarized in the $x$ direction at its waist, traveling in the $z$ direction, and incident on a spherical particle of radius $a$. Its wavelength is $\lambda$ and the wave number is $k=2 \pi / \lambda$. When the particle is located on the beam axis with coordinates $\left(0,0, z_{0}\right)$, the beam is incident on-axis. The beam shape coefficients are zero for all $m \neq \pm 1$ and the nonzero terms can be written as

$$
\begin{gathered}
g_{n, T M}^{1}=g_{n, T M}^{-1}=\frac{1}{2} g_{n}, \\
g_{n, T E}^{1}=-g_{n, T E}^{-1}=\frac{i}{2} g_{n},
\end{gathered}
$$

where in the spherical coordinates of the particle $(r, \theta, \phi) g_{n}$ is evaluated by $[10]$

$$
\begin{aligned}
g_{n}= & -\frac{2 n+1}{\pi n(n+1)}(-i)^{n+1} \int_{0}^{\pi} \int_{0}^{\infty} \frac{i k w_{0}^{2}}{2\left(z-z_{0}\right)-i k w_{0}^{2}} \exp \left[i \frac{k r^{2} \sin ^{2} \theta}{2\left(z-z_{0}\right)-i k w_{0}^{2}}\right] \exp \left(-i k z_{0}\right) \exp (i k r \cos \theta) \\
& \times\left[1-\frac{2 r \cos \theta}{2\left(z-z_{0}\right)-i k w_{0}^{2}}\right] \psi_{n}(k r) P_{n}^{1}(\cos \theta) \sin ^{2} \theta d \theta d(k r),
\end{aligned}
$$

where the Riccati-Bessel function is $\psi_{n}(k r)=k r j_{n}(k r)$ and $P_{n}^{1}$ is the associated Legendre polynomial of order $n$.

The electric field outside the sphere is the sum of the incident and scattered electric fields, namely,

$$
\begin{aligned}
\mathbf{E}= & \frac{E_{0}}{k r} \sum_{n=1}^{\infty} i^{n} \frac{2 n+1}{n(n+1)} g_{n}\left\{-\frac{i}{k r} \mathbf{e}_{r} \cos \phi\left[\psi_{n}(k r)-a_{n} \zeta_{n}^{(1)}(k r)\right] n(n+1) P_{n}^{1}(\cos \theta)+\mathbf{e}_{\theta} \cos \phi\left\{\left[\psi_{n}(k r)-b_{n} \zeta_{n}^{(1)}(k r)\right] \pi_{n}(\theta)-i\left[\psi_{n}^{\prime}(k r)\right.\right.\right. \\
& \left.\left.\left.-a_{n} \zeta_{n}^{(1)^{\prime}}(k r)\right] \tau_{n}(\theta)\right\}-\mathbf{e}_{\phi} \sin \phi\left\{\left[\psi_{n}(k r)-b_{n} \zeta_{n}^{(1)}(k r)\right] \tau_{n}(\theta)-i\left[\psi_{n}^{\prime}(k r)-a_{n} \zeta_{n}^{(1)^{\prime}}(k r)\right] \pi_{n}(\theta)\right\}\right\},
\end{aligned}
$$

where $\zeta_{n}$ are the Riccati-Hankel functions of the first and second types defined by

$$
\begin{aligned}
& \zeta_{n}^{(1)}(k r)=\psi_{n}(k r)+i \chi_{n}(k r), \\
& \zeta_{n}^{(2)}(k r)=\psi_{n}(k r)-i \chi_{n}(k r),
\end{aligned}
$$

and the Riccati-Neumann function is $\chi_{n}(k r)=k r n_{n}(k r)$.

The magnetic field at the same position outside the sphere can be evaluated by

$$
\begin{aligned}
\mathbf{H}= & \frac{E_{0}}{c k} \sum_{n=1}^{\infty} i^{n} \frac{2 n+1}{n(n+1)} g_{n}\left\{-\frac{i}{k r} \mathbf{e}_{r} \sin \phi\left[\psi_{n}(k r)\right.\right. \\
& \left.-b_{n} \zeta_{n}^{(1)}(k r)\right] n(n+1) P_{n}^{1}(\cos \theta)+\mathbf{e}_{\theta} \sin \phi\left\{\left[\psi_{n}(k r)\right.\right. \\
& \left.-a_{n} \zeta_{n}^{(1)}(k r)\right] \pi_{n}(\theta)
\end{aligned}
$$

$$
\begin{aligned}
& \left.-i\left[\psi_{n}^{\prime}(k r)-b_{n} \zeta_{n}^{(1)^{\prime}}(k r)\right] \tau_{n}(\theta)\right\}+\mathbf{e}_{\phi} \cos \phi\left\{\left[\psi_{n}(k r)\right.\right. \\
& \left.\left.\left.-a_{n} \zeta_{n}^{(1)}(k r)\right] \tau_{n}(\theta)-i\left[\psi_{n}^{\prime}(k r)-b_{n} \zeta_{n}^{(1)^{\prime}}(k r)\right] \pi_{n}(\theta)\right\}\right\} .
\end{aligned}
$$

\section{Nonabsorbing sphere}

We use the Debye series to analyze the problem where the TE partial wave scattering amplitude is written in the form of series summation [28],

$$
b_{n}=\frac{1}{2}\left[1-R_{n}^{\mathrm{I} / \mathrm{I}, T E}-\sum_{p=1}^{\infty} T_{n}^{\mathrm{I} / \mathrm{II}, T E}\left(R_{n}^{\mathrm{II} / \mathrm{II}, T E}\right)^{p-1} T_{n}^{\mathrm{I} / \mathrm{I}, T E}\right],
$$

and the TM partial wave scattering amplitude is 
$a_{n}=\frac{1}{2}\left[1-R_{n}^{\mathrm{I} / \mathrm{I}, T M}-\sum_{p=1}^{\infty} T_{n}^{\mathrm{I} / \mathrm{II}, T M}\left(R_{n}^{\mathrm{I} / \mathrm{II}, T M}\right)^{p-1} T_{n}^{\mathrm{I} / /, T M}\right]$,

where the superscript I denotes the exterior of the sphere and II denotes the interior of the sphere. The first term in the square brackets describes the diffraction of the incident wave around the sphere. The second terms, $R_{n}^{\mathrm{I} / \mathrm{I}, T E}$ and $R_{n}^{\mathrm{I} / \mathrm{I}, T M}$ are the components indicating the outgoing wave reflected from the surface of the sphere. The third term takes the series form, with each individual term $p$ representing the emerging waves after experiencing $(p-1)$ internal reflections.

For a nonabsorbing sphere, it was shown in Ref. [30] that the diffraction of high partial waves is canceled by tunneling reflection since for $n>k a$ the magnitudes of the diffracted and the externally reflected parts of the partial wave scattering amplitudes rapidly become equal and opposite and cancel, namely, $\frac{1}{2}\left(1-R_{n}^{\mathrm{I} / \mathrm{I}, T E}\right) \rightarrow 0, \frac{1}{2}\left(1-R_{n}^{\mathrm{I} / \mathrm{I}, T M}\right) \rightarrow 0$. Therefore to explore the role of diffraction, we only need to sum up to $n=k a$ to get the partial wave scattering amplitudes.

\section{a. Near-forward direction}

The following results are obtained using the method in Appendix 3 of Ref. [31]. Let $\theta$ be small so that the nearforward direction is considered. For this case we have [32]

$$
\begin{aligned}
& \pi_{n}(\theta) \approx \frac{n(n+1)}{2} J_{0}\left[\left(n+\frac{1}{2}\right) \theta\right], \\
& \tau_{n}(\theta) \approx \frac{n(n+1)}{2} J_{0}\left[\left(n+\frac{1}{2}\right) \theta\right],
\end{aligned}
$$

where $J_{0}$ is a Bessel function of the first kind.

Invoking $\mathbf{e}_{x} \approx \mathbf{e}_{\theta} \cos \phi-\mathbf{e}_{\phi} \sin \phi, \mathbf{e}_{y} \approx \mathbf{e}_{\theta} \sin \phi+\mathbf{e}_{\phi} \cos \phi$ when $\theta$ is small, substitution of Eqs. (A10) and (A11) into Eqs. (A4) and (A7) for the total fields outside the sphere gives

$$
\begin{aligned}
\mathbf{E}(r, \theta, \phi)= & \frac{E_{0}}{k r} \mathbf{e}_{x} \sum_{n=1}^{\infty} g_{n}\left(n+\frac{1}{2}\right) i^{n} J_{0}\left[\left(n+\frac{1}{2}\right) \theta\right]\left\{\left[\psi_{n}(k r)\right.\right. \\
& \left.\left.-b_{n} \zeta_{n}^{(1)}(k r)\right]-i\left[\psi_{n}^{\prime}(k r)-a_{n} \zeta_{n}^{(1)^{\prime}}(k r)\right]\right\}, \quad(\mathrm{A} 12) \\
\mathbf{H}(r, \theta, \phi)= & \frac{E_{0}}{c k r} \mathbf{e}_{y} \sum_{n=1}^{\infty} g_{n}\left(n+\frac{1}{2}\right) i^{n} J_{0}\left[\left(n+\frac{1}{2}\right) \theta\right]\left\{\left[\psi_{n}(k r)\right.\right. \\
& \left.\left.-a_{n} \zeta_{n}^{(1)}(k r)\right]-i\left[\psi_{n}^{\prime}(k r)-b_{n} \zeta_{n}^{(1)^{\prime}}(k r)\right]\right\} . \quad \text { (A1 }
\end{aligned}
$$

In the near-forward directions scattering is dominated by diffraction. Thus we substitute $\frac{1}{2}$ for $a_{n}$ and $b_{n}$ and Eqs. (A12) and (A13) become

$$
\begin{aligned}
\mathbf{E}(r, \theta, \phi)= & \frac{E_{0}}{k r} \mathbf{e}_{x} \sum_{n=1}^{\infty} g_{n}\left(n+\frac{1}{2}\right) i^{n} J_{0}\left[\left(n+\frac{1}{2}\right) \theta\right] \\
& \times\left[\left(\psi_{n}(k r)-\frac{1}{2} \zeta_{n}^{(1)}(k r)\right)\right. \\
& \left.-i\left(\psi_{n}^{\prime}(k r)-\frac{1}{2} \zeta_{n}^{(1)^{\prime}}(k r)\right)\right],
\end{aligned}
$$

$$
\begin{aligned}
H(r, \theta, \phi)= & \frac{E_{0}}{c k r} \mathbf{e}_{y} \sum_{n=1}^{\infty} g_{n}\left(n+\frac{1}{2}\right) i^{n} J_{0}\left[\left(n+\frac{1}{2}\right) \theta\right] \\
& \times\left[\left(\psi_{n}(k r)-\frac{1}{2} \zeta_{n}^{(1)}(k r)\right)\right. \\
& \left.-i\left(\psi_{n}^{\prime}(k r)-\frac{1}{2} \zeta_{n}^{(1)^{\prime}}(k r)\right)\right] .
\end{aligned}
$$

The optical stress is obtained from the fields evaluated on the surface of the sphere $(r=a)$. Since

$$
\begin{gathered}
\psi_{n}(k r)=\frac{1}{2}\left[\zeta_{n}^{(1)}(k r)+\zeta_{n}^{(2)}(k r)\right], \\
\psi_{n}^{\prime}(k r)=\frac{1}{2}\left[\zeta_{n}^{(1)^{\prime}}(k r)+\zeta_{n}^{(2)^{\prime}}(k r)\right],
\end{gathered}
$$

we have the following fields in the near-forward directions:

$$
\begin{aligned}
\mathbf{E}(a, \theta, \phi)= & \frac{E_{0}}{k a} \mathbf{e}_{x} \sum_{n=1}^{k a} g_{n}\left(n+\frac{1}{2}\right) i^{n} J_{0}\left[\left(n+\frac{1}{2}\right) \theta\right] \\
& \times\left[\frac{1}{2} \zeta_{n}^{(2)}(k a)-\frac{1}{2} i \zeta_{n}^{(2)^{\prime}}(k a)\right], \\
\mathbf{H}(a, \theta, \phi)= & \frac{E_{0}}{c k a} \mathbf{e}_{y} \sum_{n=1}^{k a} g_{n}\left(n+\frac{1}{2}\right) i^{n} J_{0}\left[\left(n+\frac{1}{2}\right) \theta\right] \\
& \times\left[\frac{1}{2} \zeta_{n}^{(2)}(k a)-\frac{1}{2} i \zeta_{n}^{(2)^{\prime}}(k a)\right] .
\end{aligned}
$$

For a sphere of large size $\alpha=k a$, defining $\gamma=\left(n+\frac{1}{2}\right) / \alpha$, we have the following asymptotic relationship for $n+\frac{1}{2}<k a$ [33]:

$$
\begin{aligned}
\zeta_{n}^{(1)}(\alpha) \approx & \frac{1}{\left(1-\gamma^{2}\right)^{1 / 4}} \\
& \times \exp \left\{i\left[x \sqrt{1-\gamma^{2}}-\left(n+\frac{1}{2}\right) \arcsin \sqrt{1-\gamma^{2}}-\frac{\pi}{4}\right]\right\}, \\
\zeta_{n}^{(2)}(\alpha) \approx & \frac{1}{\left(1-\gamma^{2}\right)^{1 / 4}} \exp \left\{-i\left[x \sqrt{1-\gamma^{2}}\right.\right. \\
& \left.\left.-\left(n+\frac{1}{2}\right) \arcsin \sqrt{1-\gamma^{2}}-\frac{\pi}{4}\right]\right\} . \quad \text { (A21) }
\end{aligned}
$$

For the derivative we have

$$
\begin{gathered}
\zeta_{n}^{(1)^{\prime}}(\alpha) \approx i \sqrt{1-\gamma^{2}} \zeta_{n}^{(1)}(\alpha), \\
\zeta_{n}^{(2)}(\alpha) \approx-i \sqrt{1-\gamma^{2}} \zeta_{n}^{(2)}(\alpha) .
\end{gathered}
$$

Thus

$$
\begin{aligned}
& \zeta_{n}^{(1)}(\alpha)+i \zeta_{n}^{(1)^{\prime}}(\alpha)=\left(1-\sqrt{1-\gamma^{2}}\right) \zeta_{n}^{(1)}(\alpha), \\
& \zeta_{n}^{(2)}(\alpha)-i \zeta_{n}^{(2))^{\prime}}(\alpha)=\left(1-\sqrt{1-\gamma^{2}}\right) \zeta_{n}^{(2)}(\alpha) .
\end{aligned}
$$

When substituted into Eqs. (A18) and (A19), Eqs. (A24) and (A25) stay small until $\gamma \rightarrow 1$ or $n+\frac{1}{2} \rightarrow k a$. Thus, the dif- 
fracted field of the partial waves with $1 \leq n \leq k a$ approximately cancels the incident field and produces the deep shadow region [31]. When $n+\frac{1}{2}$ approaches $k a$, the Bessel function $J_{0}$ in Eqs. (A18) and (A19) becomes small for large $k a$. Therefore no essential contribution is made by diffraction to the stress on the shadowed surface.

\section{b. Near-backward direction}

Let $\theta=\pi-\xi$, where $\xi$ is small so that the near-backward direction is considered. For this case [34],

$$
\begin{gathered}
\pi_{n}(\theta) \approx \frac{n(n+1)}{2}(-1)(-1)^{n} J_{0}\left[\left(n+\frac{1}{2}\right) \xi\right], \\
\tau_{n}(\theta) \approx \frac{n(n+1)}{2}(-1)^{n} J_{0}\left[\left(n+\frac{1}{2}\right) \xi\right] .
\end{gathered}
$$

Neglecting the radial component in the fields and the partial waves larger than $k a$, invoking $\mathbf{e}_{x} \approx-\mathbf{e}_{\theta} \cos \phi-\mathbf{e}_{\phi} \sin \phi$, $\mathbf{e}_{y} \approx-\mathbf{e}_{\theta} \sin \phi+\mathbf{e}_{\phi} \cos \phi$ when $\theta$ approaches $\pi$, substitution of Eqs. (A26) and (A27) into Eqs. (A4) and (A7) for the total fields outside the sphere gives

$$
\begin{aligned}
\mathbf{E}(r, \pi-\xi, \phi)= & \frac{E_{0}}{k r} \mathbf{e}_{x} \sum_{n=1}^{\infty} g_{n}\left(n+\frac{1}{2}\right)(-i)^{n} J_{0}\left[\left(n+\frac{1}{2}\right) \xi\right] \\
& \times\left\{\left[\psi_{n}(k r)-b_{n} \zeta_{n}^{(1)}(k r)\right]+i\left[\psi_{n}^{\prime}(k r)\right.\right. \\
& \left.\left.-a_{n} \zeta_{n}^{(1)^{\prime}}(k r)\right]\right\}, \\
\mathbf{H}(r, \pi-\xi, \phi)= & \frac{E_{0}}{c k r} \mathbf{e}_{y} \sum_{n=1}^{\infty} g_{n}\left(n+\frac{1}{2}\right)(-i)^{n} J_{0}\left[\left(n+\frac{1}{2}\right) \xi\right] \\
& \times\left\{\left[\psi_{n}(k r)-a_{n} \zeta_{n}^{(1)}(k r)\right]+i\left[\psi_{n}^{\prime}(k r)\right.\right. \\
& \left.\left.-b_{n} \zeta_{n}^{(1)^{\prime}}(k r)\right]\right\} .
\end{aligned}
$$

Then we substitute $\frac{1}{2}$ for $a_{n}$ and $b_{n}$ so as to look at the contributions of the incident beam and diffraction, and Eqs. (A28) and (A29) become

$$
\begin{aligned}
\mathbf{E}(r, \pi-\xi, \phi)= & \frac{E_{0}}{k r} \mathbf{e}_{x} \sum_{n=1}^{\infty} g_{n}\left(n+\frac{1}{2}\right)(-i)^{n} J_{0}\left[\left(n+\frac{1}{2}\right) \xi\right] \\
& \times\left[\left(\psi_{n}(k r)-\frac{1}{2} \zeta_{n}^{(1)}(k r)\right)\right. \\
& \left.+i\left(\psi_{n}^{\prime}(k r)-\frac{1}{2} \zeta_{n}^{(1)^{\prime}}(k r)\right)\right], \\
\mathbf{H}(r, \pi-\xi, \phi)= & \frac{E_{0}}{c k r} \mathbf{e}_{y} \sum_{n=1}^{\infty} g_{n}\left(n+\frac{1}{2}\right)(-i)^{n} \\
& \times J_{0}\left[\left(n+\frac{1}{2}\right) \xi\right]\left[\left(\psi_{n}(k r)-\frac{1}{2} \zeta_{n}^{(1)}(k r)\right)\right. \\
& \left.+i\left(\psi_{n}^{\prime}(k r)-\frac{1}{2} \zeta_{n}^{(1)^{\prime}}(k r)\right)\right] .
\end{aligned}
$$

When we evaluate the contribution of diffraction alone, only the second term in each parentheses in the square brackets is included (the first term is the incident beam) so that the following expressions are obtained in the near-backward directions:

$$
\begin{aligned}
\mathbf{E}^{\text {diffracted }}(a, \pi-\xi, \phi)= & -\frac{E_{0}}{2 k a} \mathbf{e}_{x} \sum_{n=1}^{k a} g_{n}\left(n+\frac{1}{2}\right)(-i)^{n} \\
& \times J_{0}\left[\left(n+\frac{1}{2}\right) \xi\right]\left[\zeta_{n}^{(1)}(k a)+i \zeta_{n}^{(1)^{\prime}}(k a)\right], \\
\mathbf{H}^{\text {diffracted }}(a, \pi-\xi, \phi)= & -\frac{E_{0}}{2 c k a} \mathbf{e}_{y} \sum_{n=1}^{k a} g_{n}\left(n+\frac{1}{2}\right)(-i)^{n} \\
& \times J_{0}\left[\left(n+\frac{1}{2}\right) \xi\right]\left[\zeta_{n}^{(1)}(k a)+i \zeta_{n}^{(1)^{\prime}}(k a)\right] .
\end{aligned}
$$

Invoking asymptotic relation (A24), we find that the contribution of diffraction stays negligible until $n+\frac{1}{2}$ is close to $k a$.

\section{Impenetrable sphere}

For an impenetrable sphere the emerging waves experiencing $(p-1)$ internal reflections do not occur. Therefore the TE and TM partial wave scattering amplitudes become

$$
\begin{aligned}
& b_{n}=\frac{1}{2}\left[1-R_{n}^{\mathrm{I} / \mathrm{I}, T E}\right], \\
& a_{n}=\frac{1}{2}\left[1-R_{n}^{\mathrm{I} / \mathrm{I}, T M}\right],
\end{aligned}
$$

where

$$
\begin{gathered}
R_{n}^{\mathrm{I} / \mathrm{I}, T E}=-\frac{\zeta_{n}^{(2)}(k a)}{\zeta_{n}^{(1)}(k a)}, \\
R_{n}^{\mathrm{I} / \mathrm{I}, T M}=-\frac{\zeta_{n}^{(2)^{\prime}}(k a)}{\zeta_{n}^{(1)^{\prime}}(k a)} .
\end{gathered}
$$

The TE and TM partial wave scattering amplitudes can be rewritten as

$$
\begin{aligned}
& b_{n}=\frac{1}{2}\left[1-\frac{1+i \frac{j_{n}(k a)}{n_{n}(k a)}}{1-i \frac{j_{n}(k a)}{n_{n}(k a)}}\right], \\
& a_{n}=\frac{1}{2}\left[1-\frac{1+i \frac{j_{n}^{\prime}(k a)}{n_{n}^{\prime}(k a)}}{1-i \frac{j_{n}^{\prime}(k a)}{n_{n}^{\prime}(k a)}}\right] .
\end{aligned}
$$


Notice that if $n>k a, j_{n}(k a)$ rapidly goes to zero and $n_{n}(k a)$ rapidly goes to infinity. Then the reflected amplitude cancels the diffracted amplitude and $a_{n} \rightarrow 0$, $b_{n} \rightarrow 0$, terminating the Mie sum. For $1 \leq n \leq k a$, it can be proved in the same way as for the nonabsorbing sphere that the diffracted wave is canceled by incident wave in the near-forward directions and negligible in the near-backward directions.
[1] A. Ashkin, Optical Trapping and Manipulation of Neutral Particles Using Lasers: A Reprint Volume with Commentaries (World Scientific, Singapore, 2006).

[2] J. Guck, R. Ananthakrishnan, T. J. Moon, C. C. Cunningham, and J. Käs, Phys. Rev. Lett. 84, 5451 (2000).

[3] E. Higurashi, H. Utika, H. Tanaka, and O. Ohguchi, Appl. Phys. Lett. 64, 2209 (1994).

[4] R. C. Gauthier, Appl. Phys. Lett. 69, 2015 (1996).

[5] F. Borghese, P. Denti, R. Saija, M. A. Iatì, and O. M. Maragò, Phys. Rev. Lett. 100, 163903 (2008).

[6] K. C. Neuman and S. M. Block, Rev. Sci. Instrum. 75, 2787 (2004).

[7] H. C. van de Hulst, Light Scattering by Small Particles (Dover, New York, 1981), Chap. 9.

[8] P. L. Marston and J. H. Crichton, Phys. Rev. A 30, 2508 (1984).

[9] G. Gouesbet and G. Gréhan, J. Opt. (Paris) 13, 97 (1982).

[10] G. Gouesbet, B. Maheu, and G. Gréhan, J. Opt. Soc. Am. A 5, 1427 (1988).

[11] H. Polaert, G. Gréhan, and G. Gouesbet, Opt. Commun. 155, 169 (1998).

[12] J. P. Barton, D. R. Alexander, and S. A. Schaub, J. Appl. Phys. 66, 4594 (1989).

[13] J. S. Kim and S. S. Lee, J. Opt. Soc. Am. 73, 303 (1983).

[14] S. Chang and S. S. Lee, J. Opt. Soc. Am. B 2, 1853 (1985).

[15] J. Guck, R. Ananthakrishnan, H. Mahmood, T. J. Moon, C. Cunningham, and J. Käs, Biophys. J. 81, 767 (2001).

[16] G. Gouesbet, G. Gréhan, and B. Maheu, Appl. Opt. 27, 4874 (1988).
[17] J. D. Jackson, Classical Electrodynamics, 2nd ed. (Wiley, New York, 1975).

[18] J. A. Stratton, Electromagnetic Theory (McGraw-Hill, New York, 1941).

[19] F. Xu, K. F. Ren, and X. Cai, Appl. Opt. 45, 4990 (2006).

[20] J. P. Barton and D. R. Alexander, J. Appl. Phys. 66, 2800 (1989).

[21] G. M. Hale and M. R. Querry, Appl. Opt. 12, 555 (1973).

[22] M. V. Berry and C. Upstill, Prog. Opt. 18, 257 (1980).

[23] V. Srivastava and M. A. Jarzembski, Opt. Lett. 16, 126 (1991).

[24] H. M. Nussenzveig, Ann. Phys. (N.Y.) 34, 23 (1965).

[25] F. Xu, J. A. Lock, G. Gouesbet, and C. Tropea, Phys. Rev. A 78, 013843 (2008).

[26] G. Gouesbet, J. A. Lock, and G. Gréhan, Appl. Opt. 34, 2133 (1995).

[27] E. Evans and Y. C. Fung, Microvasc. Res. 4, 335 (1972).

[28] E. A. Hovenac and J. A. Lock, J. Opt. Soc. Am. A 9, 781 (1992).

[29] J. A. Lock and E. A. Hovenac, J. Opt. Soc. Am. A 8, 1541 (1991).

[30] J. A. Lock and E. A. Hovenac, Am. J. Phys. 61, 698 (1993).

[31] L. Brillouin, J. Appl. Phys. 20, 1110 (1949).

[32] H. C. van de Hulst, in Light Scattering by Small Particles (Ref. [7]), p. 209.

[33] Handbook of Mathematical Functions, edited by M. Abramowitz and I. A. Stegun (Dover, New York, 1964), p. 366.

[34] H. C. van de Hulst, in Light Scattering by Small Particles (Ref. [7]), p. 253. 